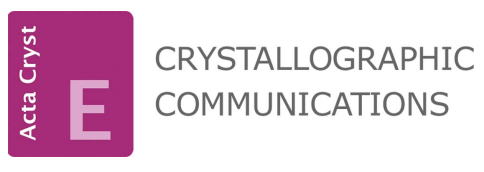

ISSN 2056-9890
Received 13 February 2017

Accepted 19 February 2017

Edited by M. Weil, Vienna University of

Technology, Austria

Keywords: crystal structure; tetrakis-thioethersubstituted pyrazines; silver(I) nitrate; metalorganic chain (MOC); metal-organic network (MON); metal-organic framework (MOF); $\mathrm{C}-$ $\mathrm{H} \cdots \mathrm{O}$ and $\mathrm{C}-\mathrm{H} \cdots \mathrm{S}$ hydrogen bonds.

CCDC references: 1533573; 1533572; 1533571

Supporting information: this article has supporting information at journals.iucr.org/e

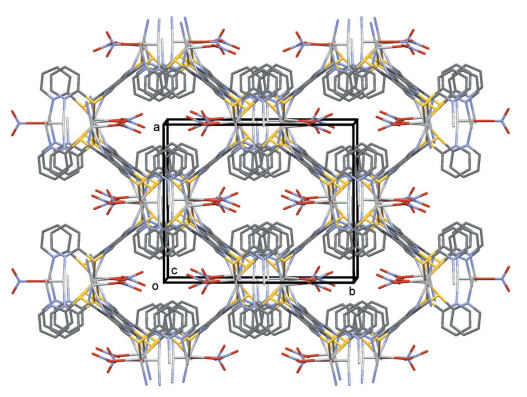

OPEN $\odot$ ACCESS

\section{Silver(I) nitrate complexes of three tetrakis-thio- ether-substituted pyrazine ligands: metal-organic chain, network and framework structures}

\author{
Tokouré Assoumatine $^{\mathrm{a}}$ and Helen Stoeckli-Evans ${ }^{\mathrm{b}}$ *
} a'CanAm Bioresearch Inc., 9-1250 Waverley Street, Winnipeg, Manitoba R3T 6C6, Canada, and ${ }^{\mathbf{b}}$ Institute of Physics,
University of Neuchâtel, rue Emile-Argand11,CH-2000 Neuchâtel, Switzerland. *Correspondence e-mail:
helen.stoeckli-evans@unine.ch

The reaction of the ligand 2,3,5,6-tetrakis[(methylsulfanyl)methyl]pyrazine (L1) with silver(I) nitrate led to $\left\{\left[\mathrm{Ag}\left(\mathrm{C}_{12} \mathrm{H}_{20} \mathrm{~N}_{2} \mathrm{~S}_{4}\right)\right]\left(\mathrm{NO}_{3}\right)\right\}_{n}$, (I), catena-poly[[sil$\operatorname{ver}(\mathrm{I})-\mu-2,3,5,6$-tetrakis[(methylsulfanyl)methyl]pyrazine] nitrate], a compound with a metal-organic chain structure. The asymmetric unit is composed of two half ligands, located about inversion centres, with one ligand coordinating to the silver atoms in a bis-tridentate manner and the other in a bis-bidentate manner. The charge on the metal atom is compensated for by a free nitrate anion. Hence, the silver atom has a fivefold $\mathrm{S}_{3} \mathrm{~N}_{2}$ coordination sphere. The reaction of the ligand 2,3,5,6-tetrakis[(phenylsulfanyl)methyl]pyrazine (L2) with silver(I) nitrate, led to $\left[\mathrm{Ag}_{2}\left(\mathrm{NO}_{3}\right)_{2}\left(\mathrm{C}_{32} \mathrm{H}_{28} \mathrm{~N}_{2} \mathrm{~S}_{4}\right)\right]_{n}$, (II), poly[di- $\mu$-nitrato-bis $\{\mu$-2,3,5,6tetrakis[(phenylsulfanyl)methyl]pyrazine\}disilver], a compound with a metalorganic network structure. The asymmetric unit is composed of half a ligand, located about an inversion centre, that coordinates to the silver atoms in a bistridentate manner. The nitrate anion coordinates to the silver atom in a bidentate/monodentate manner, bridging the silver atoms, which therefore have a sixfold $\mathrm{S}_{2} \mathrm{NO}_{3}$ coordination sphere. The reaction of the ligand 2,3,5,6tetrakis[(pyridin-2-ylsulfanyl)methyl]pyrazine (L3) with silver(I) nitrate led to $\left[\mathrm{Ag}_{3}\left(\mathrm{NO}_{3}\right)_{3}\left(\mathrm{C}_{28} \mathrm{H}_{24} \mathrm{~N}_{6} \mathrm{~S}_{4}\right)\right]_{n}$, (III), poly[trinitrato $\left\{\mu_{6}-2,3,5,6\right.$-tetrakis[(pyridin-2ylsulfanyl)methyl]pyrazine trisilver(I)], a compound with a metal-organic framework structure. The asymmetric unit is composed of half a ligand, located about an inversion centre, that coordinates to the silver atoms in a bis-tridentate manner. One pyridine $\mathrm{N}$ atom bridges the monomeric units, so forming a chain structure. Two nitrate $\mathrm{O}$ atoms also coordinate to this silver atom, hence it has a sixfold $\mathrm{S}_{2} \mathrm{~N}_{2} \mathrm{O}_{2}$ coordination sphere. The chains are linked via a second silver atom, located on a twofold rotation axis, coordinated by the second pyridine $\mathrm{N}$ atom. A second nitrate anion, also lying about the twofold rotation axis, coordinates to this silver atom via an $\mathrm{Ag}-\mathrm{O}$ bond, hence this second silver atom has a threefold $\mathrm{N}_{2} \mathrm{O}$ coordination sphere. In the crystal of (I), the nitrate anion plays an essential role in forming $\mathrm{C}-\mathrm{H}$... O hydrogen bonds that link the metal-organic chains to form a three-dimensional supramolecular structure. In the crystal of (II), the metal-organic networks (lying parallel to the $b c$ plane) stack up the $a$-axis direction but there are no significant intermolecular interactions present between the layers. In the crystal of (III), there are a number of $\mathrm{C}-\mathrm{H} \cdots \mathrm{O}$ hydrogen bonds present within the metal-organic framework. The role of the nitrate anion in the formation of the coordination polymers is also examined.

\section{Chemical context}

A series of tetrakis-thioether pyrazine ligands have been prepared in order to study their coordination behaviour with various transition metals (Assoumatine, 1999). The ligands 2,3,5,6-tetrakis[(methylsulfanyl)methyl]pyrazine (L1), 2,3,5,6- 
tetrakis[(phenylsulfanyl)methyl]pyrazine (L2) and 2,3,5,6tetrakis[(pyridin-2-ylsulfanyl)methyl]pyrazine (L3), were synthesized by the reaction of 2,3,5,6-tetrakis(bromomethyl)pyrazine (Assoumatine \& Stoeckli-Evans, 2014b), with the appropriate 2-mercapto derivative. Their crystal structures and syntheses have been reported previously: L1 (Assoumatine \& Stoeckli-Evans, 2014a), L2 (Assoumatine et al., 2007) and $\mathbf{L 3}$ (Assoumatine \& Stoeckli-Evans, 2016). The reaction of similar ligands with various silver(I) salts have also resulted in the formation of coordination polymers. For example, $2-\{[$ (pyridin-4-ylmethyl)sulfanyl]methyl\}pyrazine (Black \& Hanton, 2007) led to metal-organic frameworks, while ligands 2,3-bis \{[(pyridin-2-ylmethyl)sulfanyl]methyl\}pyrazine (Caradoc-Davies \& Hanton, 2001) and 2,5-bis \{[(pyridin-2-ylmethyl)sulfanyl]methyl\}pyrazine (Caradoc-Davies et al., 2001) both resulted in compounds with metal-organic chains.<smiles>CSCc1nc(CSC)c(CSC)nc1CSC</smiles>

L1<smiles>c1ccc(SCc2nc(CSc3ccccc3)c(CSc3ccccc3)nc2CSc2ccccc2)cc1</smiles>

L2<smiles>c1ccc(SCc2nc(CSc3ccccn3)c(CSc3ccccn3)nc2CSc2ccccn2)nc1</smiles>

L3
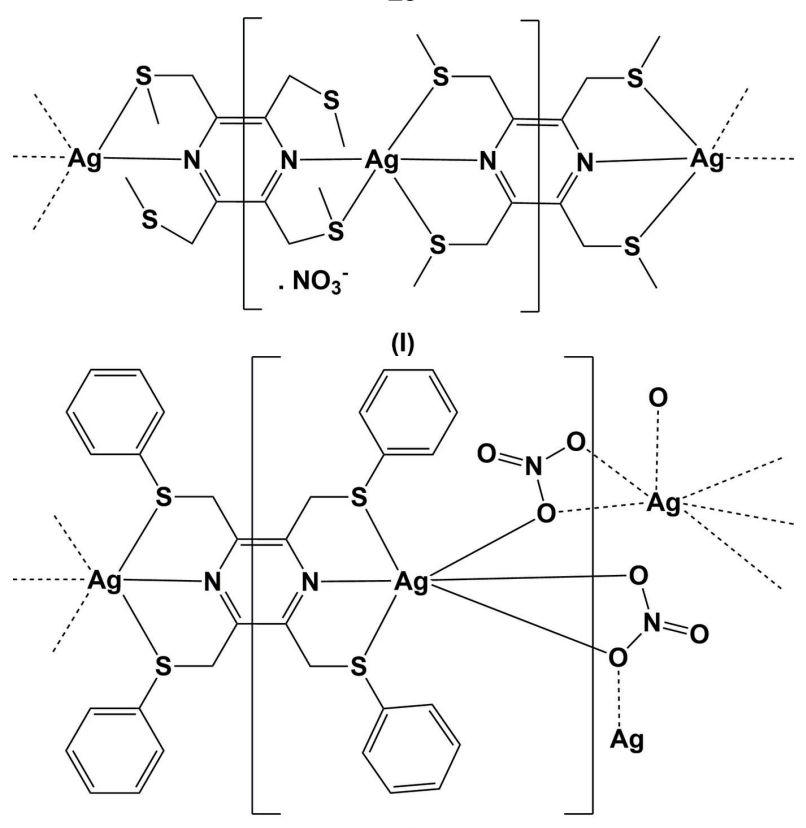

(II)

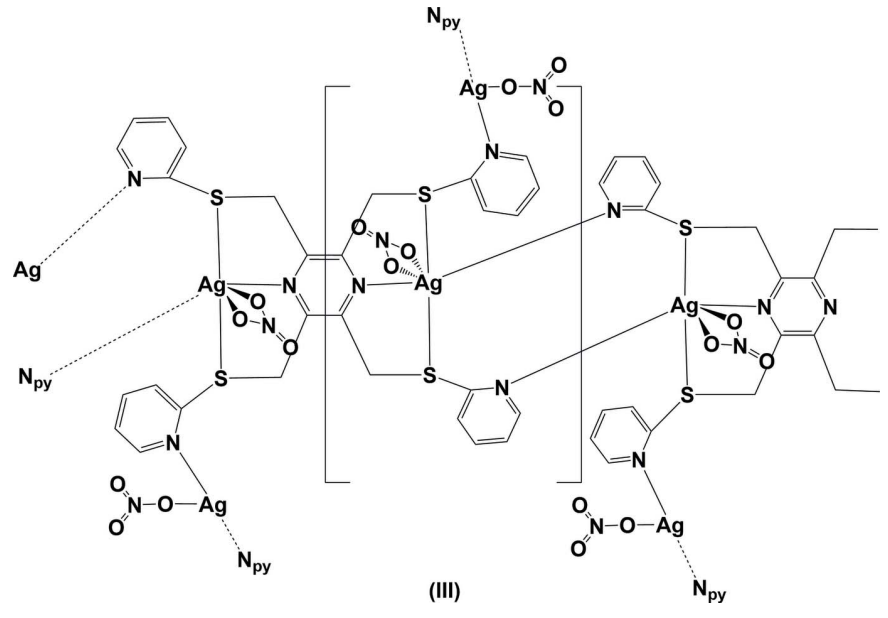

\section{Structural commentary}

The reaction of the ligand 2,3,5,6-tetrakis[(methylsulfanyl)methyl]pyrazine (L1) with silver(I) nitrate, led to the formation of a metal-organic chain (MOC) structure, (I) (Fig. 1). Selected bond lengths and angles involving the Ag1 atom are given in Table 1 . The asymmetric unit is composed of two half ligands, located about inversion centres, with one ligand coordinating to the silver atom in a bis-tridentate manner and the other in a bis-bidentate manner. Their pyrazine rings are almost normal to one another, making a dihedral angle of $88.6(2)^{\circ}$. The charge on the metal atom is compensated for by a free nitrate anion. The silver atom, $\mathrm{Ag} 1$, has a fivefold $\mathrm{S}_{3} \mathrm{~N}_{2}$ coordination sphere with a highly distorted shape and a $\tau_{5}$ value of $0.63\left(\tau_{5}=0\right.$ for an ideal square-pyramidal coordination sphere, and $=1$ for an ideal trigonal-pyramidal coordination sphere; Addison et al., 1984). Within the MOC structure, there are significant $\mathrm{C}-\mathrm{H} \cdots \mathrm{S}$ interactions present, involving the thioether substituent that does not coordinate to the silver atom, viz. atom S3 (Table 4 and Fig. 1).

The reaction of the ligand 2,3,5,6-tetrakis[(phenylsulfanyl)methyl]pyrazine (L2) with silver(I) nitrate, led to the

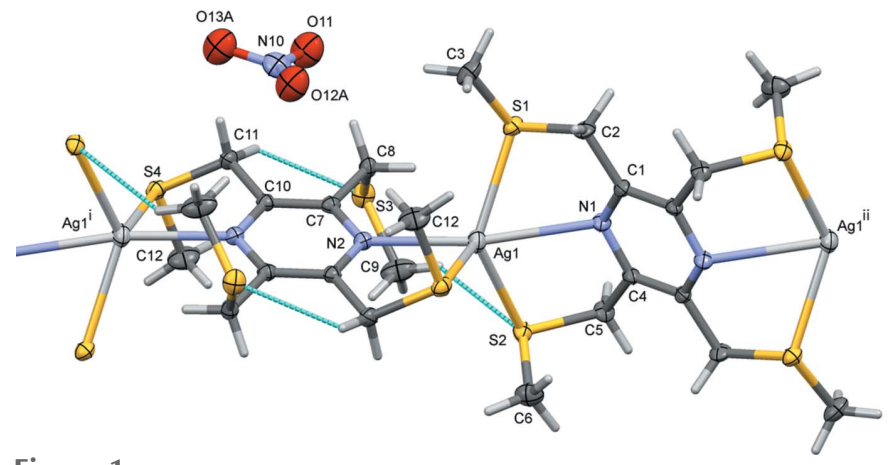

Figure 1

The molecular entities of compound (I), with atom labelling for the asymmetric unit. Unlabelled atoms are related to labelled atoms by symmetry operation (i) $=-x,-y+1,-z+1$, for the ligand involving atom $\mathrm{N} 2$, and by symmetry operation (ii) $=-x+1,-y+1,-z+2$, for the ligand involving atom N1. Displacement ellipsoids are drawn at the $50 \%$ probability level. The intramolecular $\mathrm{C}-\mathrm{H} \cdots \mathrm{S}$ contacts are shown as dashed lines (see Table 4). 
Table 1

Selected geometric parameters $\left(\AA,^{\circ}\right)$ for $(\mathrm{I})$.

\begin{tabular}{lclr}
\hline Ag1-N1 & $2.714(4)$ & Ag1-S2 & $2.5987(16)$ \\
Ag1-N2 & $2.436(5)$ & Ag1-S4 & $2.5910(15)$ \\
Ag1-S1 & $2.5895(15)$ & & \\
& & & \\
N1-Ag1-N2 & $167.75(13)$ & $\mathrm{N} 2-\mathrm{Ag} 1-\mathrm{S} 2$ & $109.60(11)$ \\
$\mathrm{N} 1-\mathrm{Ag} 1-\mathrm{S} 1$ & $64.36(9)$ & $\mathrm{N} 2-\mathrm{Ag} 1-\mathrm{S} 4^{\mathrm{i}}$ & $77.43(10)$ \\
$\mathrm{N} 1-\mathrm{Ag} 1-\mathrm{S} 2$ & $72.54(9)$ & $\mathrm{S} 1-\mathrm{Ag} 1-\mathrm{S} 2$ & $129.99(5)$ \\
$\mathrm{N} 1-\mathrm{Ag} 1-\mathrm{S} 4^{\mathrm{i}}$ & $113.79(9)$ & $\mathrm{S} 1-\mathrm{Ag} 1-\mathrm{S} 4^{\mathrm{i}}$ & $111.41(5)$ \\
$\mathrm{N} 2-\mathrm{Ag} 1-\mathrm{S} 1$ & $107.74(11)$ & $\mathrm{S} 4^{\mathrm{i}}-\mathrm{Ag} 1-\mathrm{S} 2$ & $108.26(5)$ \\
\hline
\end{tabular}

Symmetry code: (i) $-x,-y+1,-z+1$.

Table 2

Selected geometric parameters $\left(\AA{ }^{\circ}\right.$, for (II).

\begin{tabular}{lclr}
\hline $\mathrm{Ag} 1-\mathrm{N} 1$ & $2.527(4)$ & $\mathrm{Ag} 1-\mathrm{O} 1$ & $2.551(4)$ \\
$\mathrm{Ag} 1-\mathrm{S} 1$ & $2.6560(15)$ & $\mathrm{Ag} 1-\mathrm{O} 2$ & $2.507(4)$ \\
$\mathrm{Ag} 1-\mathrm{S} 2^{\mathrm{i}}$ & $2.6790(14)$ & $\mathrm{Ag} 1-\mathrm{O} 2^{\mathrm{ii}}$ & $2.539(4)$ \\
& & & \\
$\mathrm{N} 1-\mathrm{Ag} 1-\mathrm{S} 1$ & $76.40(9)$ & $\mathrm{O} 2^{\mathrm{ii}}-\mathrm{Ag} 1-\mathrm{O} 1$ & $49.56(12)$ \\
$\mathrm{N} 1-\mathrm{Ag} 1-\mathrm{S} 2^{\mathrm{i}}$ & $70.89(9)$ & $\mathrm{O} 2-\mathrm{Ag} 1-\mathrm{S} 1$ & $80.10(11)$ \\
$\mathrm{S} 1-\mathrm{Ag} 1-\mathrm{S} 2^{\mathrm{i}}$ & $146.98(4)$ & $\mathrm{O} 2^{\mathrm{ii}}-\mathrm{Ag} 1-\mathrm{S} 1$ & $101.67(11)$ \\
$\mathrm{O} 2-\mathrm{Ag} 1-\mathrm{N} 1$ & $112.54(12)$ & $\mathrm{O} 1-\mathrm{Ag} 1-\mathrm{S} 1$ & $120.09(11)$ \\
$\mathrm{O} 2-\mathrm{Ag} 1-\mathrm{O} 2^{\mathrm{ii}}$ & $117.32(8)$ & $\mathrm{O} 2-\mathrm{Ag} 1-\mathrm{S} 2^{\mathrm{i}}$ & $116.46(10)$ \\
$\mathrm{N} 1-\mathrm{Ag} 1-\mathrm{O} 2^{\mathrm{ii}}$ & $128.98(12)$ & $\mathrm{O} 2^{\mathrm{ii}}-\mathrm{Ag} 1-\mathrm{S}^{\mathrm{i}}$ & $95.47(11)$ \\
$\mathrm{O} 2-\mathrm{Ag} 1-\mathrm{O} 1$ & $75.15(13)$ & $\mathrm{O} 1-\mathrm{Ag} 1-\mathrm{S} 2^{\mathrm{i}}$ & $92.47(11)$ \\
$\mathrm{N} 1-\mathrm{Ag} 1-\mathrm{O} 1$ & $163.34(14)$ & & \\
\hline
\end{tabular}

Symmetry codes: (i) $-x+2,-y+2,-z+1$; (ii) $x,-y+\frac{3}{2}, z+\frac{1}{2}$.

formation of a metal-organic network (MON) structure, (II) (Fig. 2). Selected bond lengths and angles involving atom Ag1 are given in Table 2 . The asymmetric unit is composed of half a ligand, located about an inversion centre, a silver atom and a nitrate anion. The ligand coordinates to the silver atoms in a

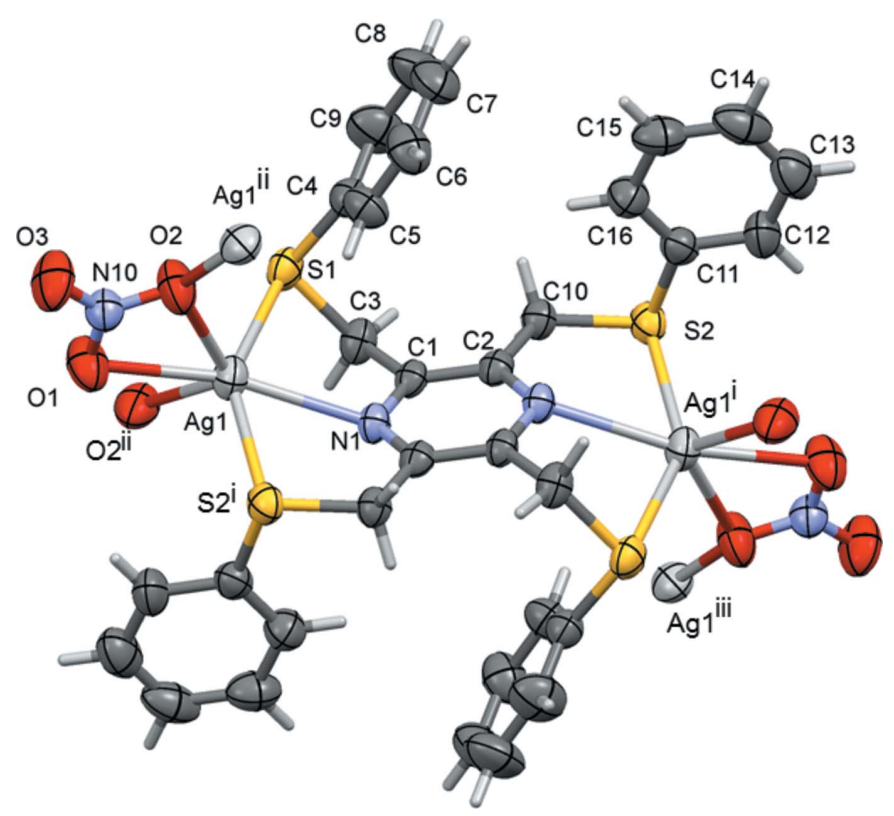

Figure 2

The molecular entities of compound (II), with atom labelling for the asymmetric unit. For the ligand, unlabelled atoms are related to the labelled atoms by symmetry operation (i) $-x+2,-y+2,-z+1$; other symmetry codes are (ii) $x,-y+\frac{3}{2}, z+\frac{1}{2}$; (iii) $-x+2, y+\frac{1}{2},-z+\frac{1}{2}$. Displacement ellipsoids are drawn at the $50 \%$ probability level.
Table 3

Selected geometric parameters $\left(\AA,^{\circ}\right)$ for (III).

\begin{tabular}{lclr}
\hline $\mathrm{Ag} 1-\mathrm{N} 1$ & $2.578(3)$ & $\mathrm{Ag} 1-\mathrm{O} 11$ & $2.700(5)$ \\
$\mathrm{Ag} 1-\mathrm{N} 2^{\mathrm{i}}$ & $2.267(3)$ & $\mathrm{Ag} 1-\mathrm{O} 13$ & $2.752(5)$ \\
$\mathrm{Ag} 1-\mathrm{S} 1$ & $2.7943(13)$ & $\mathrm{Ag} 2-\mathrm{N} 3$ & $2.208(3)$ \\
$\mathrm{Ag} 1-\mathrm{S} 2^{\mathrm{ii}}$ & $2.6010(11)$ & $\mathrm{Ag} 2-\mathrm{O} 21$ & $2.567(5)$ \\
& & & \\
$\mathrm{N} 1-\mathrm{Ag} 1-\mathrm{N} 2^{\mathrm{i}}$ & $155.31(11)$ & $\mathrm{S}^{\mathrm{ii}}-\mathrm{Ag} 1-\mathrm{O} 13$ & $120.26(10)$ \\
$\mathrm{S} 1-\mathrm{Ag} 1-\mathrm{S} 2^{\mathrm{ii}}$ & $122.71(3)$ & $\mathrm{O} 11-\mathrm{Ag} 1-\mathrm{N} 1$ & $73.76(11)$ \\
$\mathrm{S} 1-\mathrm{Ag} 1-\mathrm{N} 1$ & $68.98(7)$ & $\mathrm{O} 11-\mathrm{Ag} 1-\mathrm{N} 2^{\mathrm{i}}$ & $99.33(12)$ \\
$\mathrm{S} 1-\mathrm{Ag} 1-\mathrm{N} 2^{\mathrm{i}}$ & $96.92(8)$ & $\mathrm{O} 13-\mathrm{Ag} 1-\mathrm{N} 1$ & $69.73(11)$ \\
$\mathrm{S}^{\mathrm{ii}}-\mathrm{Ag} 1-\mathrm{N} 1$ & $70.29(7)$ & $\mathrm{O} 13-\mathrm{Ag} 1-\mathrm{N} 2^{\mathrm{i}}$ & $88.28(12)$ \\
$\mathrm{S} 2^{\mathrm{ii}}-\mathrm{Ag} 1-\mathrm{N} 2^{\mathrm{i}}$ & $133.03(8)$ & $\mathrm{O} 11-\mathrm{Ag} 1-\mathrm{O} 13$ & $45.99(14)$ \\
$\mathrm{S} 1-\mathrm{Ag} 1-\mathrm{O} 11$ & $122.18(10)$ & $\mathrm{N} 3-\mathrm{Ag} 2-\mathrm{N} 3^{\mathrm{iii}}$ & $175.41(12)$ \\
$\mathrm{S} 1-\mathrm{Ag} 1-\mathrm{O} 13$ & $79.78(10)$ & $\mathrm{O} 21-\mathrm{Ag} 2-\mathrm{N} 3$ & $92.30(9)$ \\
$\mathrm{S} 2^{\mathrm{ii}}-\mathrm{Ag} 1-\mathrm{O} 11$ & $81.18(10)$ & & \\
\hline
\end{tabular}

Symmetry codes: (i) $-x,-y+1,-z$; (ii) $-x+\frac{1}{2},-y+\frac{1}{2},-z$; (iii) $-x+1, y,-z+\frac{1}{2}$.

bis-tridentate manner. The nitrate anion coordinates to the silver atom in a bidentate/monodentate manner, bridging the silver atoms, which therefore have a sixfold $\mathrm{S}_{2} \mathrm{NO}_{3}$ coordination sphere, best described as a highly distorted octahedron (Table 2).

The reaction of the ligand 2,3,5,6-tetrakis[(pyridin-2-ylsulfanyl)methyl]pyrazine (L3) with silver(I) nitrate, led to the formation of a metal-organic framework (MOF) structure, (III) (Fig. 3). Selected bond lengths and angles involving atoms Ag1 and Ag2 are given in Table 3. The asymmetric unit is composed of half a ligand, located about an inversion centre, a silver atom and a nitrate anion, plus half a second $\mathrm{AgNO}_{3}$ unit located about a twofold rotation axis. The organic ligand coordinates to the silver atoms (Ag1), in a bis-tridentate manner. One pyridine $\mathrm{N}$ atom, N2, bridges the monomeric units, so forming a chain structure along the $b$-axis direction. The nitrate $\mathrm{O}$ atoms, $\mathrm{O} 11$ and $\mathrm{O} 13$, coordinate to silver atom $\mathrm{Ag} 1$, hence it has a highly distorted octahedral $\mathrm{S}_{2} \mathrm{~N}_{2} \mathrm{O}_{2}$ coordination sphere (Table 3). The chains are linked via a second silver atom, $\mathrm{Ag} 2$, located on a twofold rotation axis, coordinated by the second pyridine $\mathrm{N}$ atom, N3. A second nitrate anion, also lying about the twofold rotation axis,

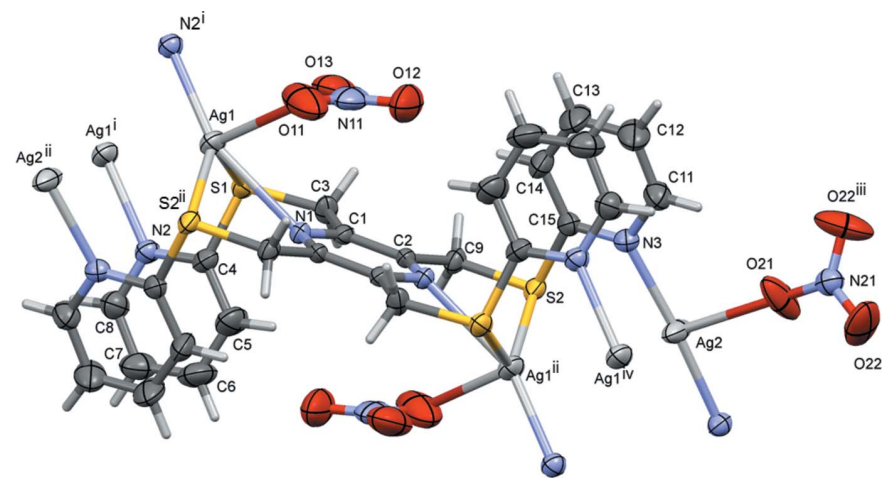

Figure 3

The molecular entities of compound (III), with atom labelling for the asymmetric unit. For the ligand, unlabelled atoms are related to the labelled atoms by symmetry operation (ii) $-x+\frac{1}{2},-y+\frac{1}{2},-z$; other symmetry codes are (i) $-x,-y+1,-z$; (iii) $-x+1, y,-z+\frac{1}{2}$; (iv) $x+\frac{1}{2}$, $y-\frac{1}{2}, z$. Displacement ellipsoids are drawn at the $50 \%$ probability level. 
Table 4

Hydrogen-bond geometry $\left(\AA{ }^{\circ}\right)$ for (I).

\begin{tabular}{|c|c|c|c|c|}
\hline$D-\mathrm{H} \cdots A$ & $D-\mathrm{H}$ & $\mathrm{H} \cdots A$ & $D \cdots A$ & $D-\mathrm{H} \cdots A$ \\
\hline $\mathrm{C} 9-\mathrm{H} 9 C \cdots \mathrm{S} 2$ & 0.96 & 2.86 & $3.650(8)$ & 141 \\
\hline $\mathrm{C} 11-\mathrm{H} 11 A \cdots \mathrm{S} 3$ & 0.97 & 2.74 & $3.502(6)$ & 136 \\
\hline $\mathrm{C} 11-\mathrm{H} 11 B \cdots \mathrm{O} 13 A$ & 0.97 & 2.52 & $3.438(17)$ & 157 \\
\hline $\mathrm{C} 2-\mathrm{H} 2 A \cdots \mathrm{O} 11^{\mathrm{ii}}$ & 0.97 & 2.55 & $3.460(9)$ & 156 \\
\hline $\mathrm{C} 2-\mathrm{H} 2 B \cdots \mathrm{O} 12 A^{\mathrm{iii}}$ & 0.97 & 2.53 & $3.431(15)$ & 154 \\
\hline $\mathrm{C} 3-\mathrm{H} 3 C \cdots \mathrm{O} 12 A^{\mathrm{iii}}$ & 0.96 & 2.37 & $3.171(17)$ & 141 \\
\hline $\mathrm{C} 3-\mathrm{H} 3 C \cdots \mathrm{O} 12 B^{\mathrm{iii}}$ & 0.96 & 2.57 & $3.364(16)$ & 140 \\
\hline $\mathrm{C} 6-\mathrm{H} 6 A \cdots \mathrm{O} 13 A^{\mathrm{i}}$ & 0.96 & 2.52 & $3.375(19)$ & 149 \\
\hline $\mathrm{C} 9-\mathrm{H} 9 A \cdots \mathrm{O} 11^{\mathrm{iv}}$ & 0.96 & 2.58 & $3.503(10)$ & 162 \\
\hline
\end{tabular}

Table 5

Hydrogen-bond geometry $\left(\AA,^{\circ}\right)$ for (III).

\begin{tabular}{lllll}
\hline$D-\mathrm{H} \cdots A$ & $D-\mathrm{H}$ & $\mathrm{H} \cdots A$ & $D \cdots A$ & $D-\mathrm{H} \cdots A$ \\
\hline $\mathrm{C} 11-\mathrm{H} 11 \cdots \mathrm{O} 21$ & 0.94 & 2.57 & $3.287(5)$ & 133 \\
$\mathrm{C} 3-\mathrm{H} 3 B \cdots \mathrm{O} 21^{\text {iv }}$ & 0.98 & 2.40 & $3.253(4)$ & 145 \\
$\mathrm{C} 3-\mathrm{H} 3 B \cdots \mathrm{O} 22^{\text {iv }}$ & 0.98 & 2.49 & $3.420(6)$ & 158 \\
$\mathrm{C}^{2}-\mathrm{H} 7 \cdots \mathrm{O} 13^{\mathrm{v}}$ & 0.94 & 2.51 & $3.268(6)$ & 138 \\
$\mathrm{C} 9-\mathrm{H} 9 A \cdots \mathrm{O} 22^{\text {iv }}$ & 0.98 & 2.32 & $3.291(6)$ & 171 \\
$\mathrm{C} 12-\mathrm{H} 12 \cdots \mathrm{O} 11^{\text {vi }}$ & 0.94 & 2.51 & $3.310(7)$ & 142 \\
$\mathrm{C} 14-\mathrm{H} 14 \cdots \mathrm{O} 22^{\text {iv }}$ & 0.94 & 2.59 & $3.349(7)$ & 138 \\
\hline
\end{tabular}

Symmetry codes: (iv) $x-\frac{1}{2}, y+\frac{1}{2}, z$; (v) $x+\frac{1}{2}, y+\frac{1}{2}, z$; (vi) $-x,-y,-z$.

coordinates to this silver atom via an $\mathrm{Ag} 2-\mathrm{O} 21$ bond, hence silver atom $\mathrm{Ag} 2$ has a T-shaped $\mathrm{N}_{2} \mathrm{O}$ coordination sphere.

It can be seen from Tables 1-3 that the $\mathrm{Ag}-\mathrm{N}$ (pyrazine) and $\mathrm{Ag}-\mathrm{S}$ bond lengths differ considerably for the three compounds. In compound (I), the $\operatorname{Ag} 1-\mathrm{N} 2$ bond length, involving the ligand that coordinates in a bis-bidentate manner, is considerably shorter at 2.436 (5) $\AA$, compared to the Ag1-N1 bond length of 2.714 (4) $\AA$, involving the ligand that coordinates in a bis-tridentate manner. These $\mathrm{Ag}-$ $\mathrm{N}$ (pyrazine) bond lengths contrast with those for compounds (II) and (III), where both ligands coordinate in a bis-tridentate

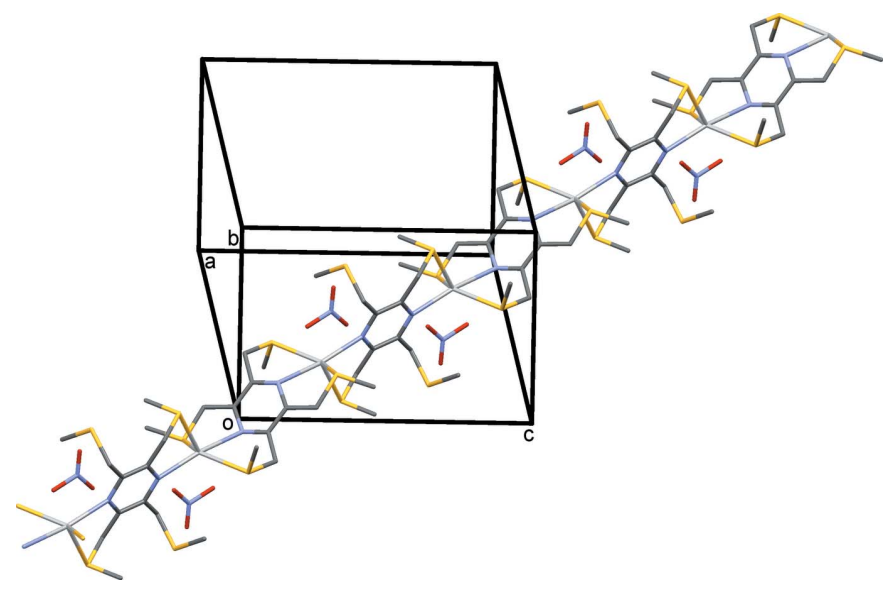

Figure 4

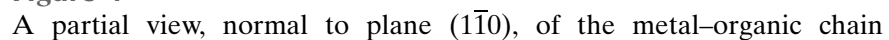
structure of compound (I). The $\mathrm{H}$ atoms have been omitted for clarity

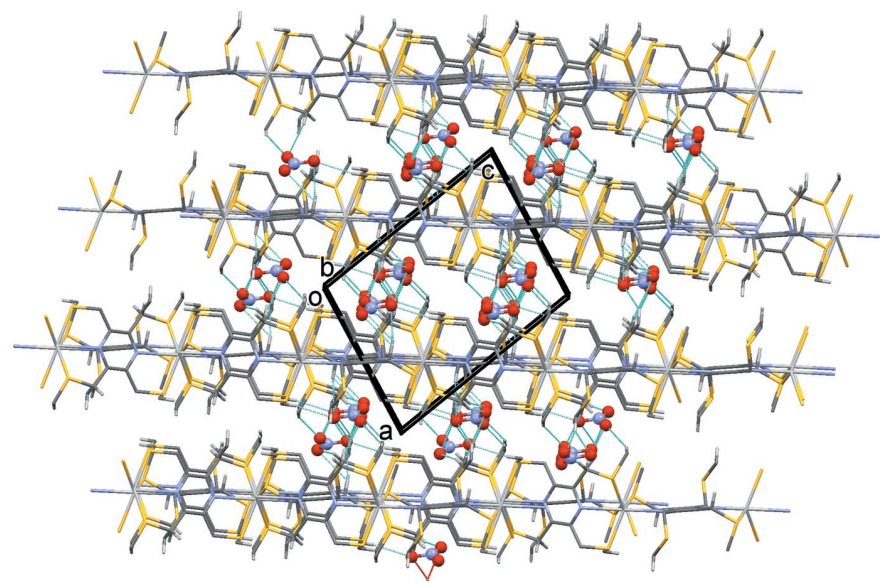

Figure 5

A view along the $b$ axis of compound (I), with emphasis on the crystal packing. Hydrogen bonds are shown as dashed lines (see Table 4), and only those $\mathrm{H}$ atoms involved in intermolecular $\mathrm{C}-\mathrm{H} \cdots \mathrm{O}$ hydrogen bonds have been included.

manner, with values of 2.527 (4) and 2.578 (3) A, respectively. The Ag1-S bond lengths in compound (I) are almost the same, varying from 2.5895 (15) to 2.5987 (16) А. These distances are shorter than those in (II), which are 2.6560 (15) and 2.6790 (14) $\AA$, but similar to bond length $\mathrm{Ag} 1-\mathrm{S} 2{ }^{\mathrm{ii}}=$ 2.6010 (11) $\AA$ in (III). The longest $\mathrm{Ag}-\mathrm{S}$ distance [2.7943 (13) $\AA$ ] is found for bond Ag1-S1 in (III). Finally, in compound (III), the two $\mathrm{Ag}-\mathrm{N}$ (pyridine) bond lengths also differ; $\mathrm{Ag} 1-\mathrm{N} 2 \mathrm{i}$ is 2.267 (3) $\AA$, while bond length $\mathrm{Ag} 2-\mathrm{N} 3$ is shorter at 2.208 (3) $\AA$ (see Table 3). Despite the large variation in the $\mathrm{Ag}-\mathrm{N}$ (pyrazine), $\mathrm{Ag}-\mathrm{S}$ or $\mathrm{Ag}-\mathrm{N}$ (pyridine) bond lengths, which perhaps indicates how flexible the ligands are, the values are within the limits observed for similar silver coordinating pyrazine, thioether or pyridine ligands, when compared to the values observed for such structures present in the Cambridge Structural Database (Groom et al., 2016). The various histograms of the bond lengths have skewed-right distributions and the values vary from 2.10 to $2.75 \AA$ for $\mathrm{Ag}-$

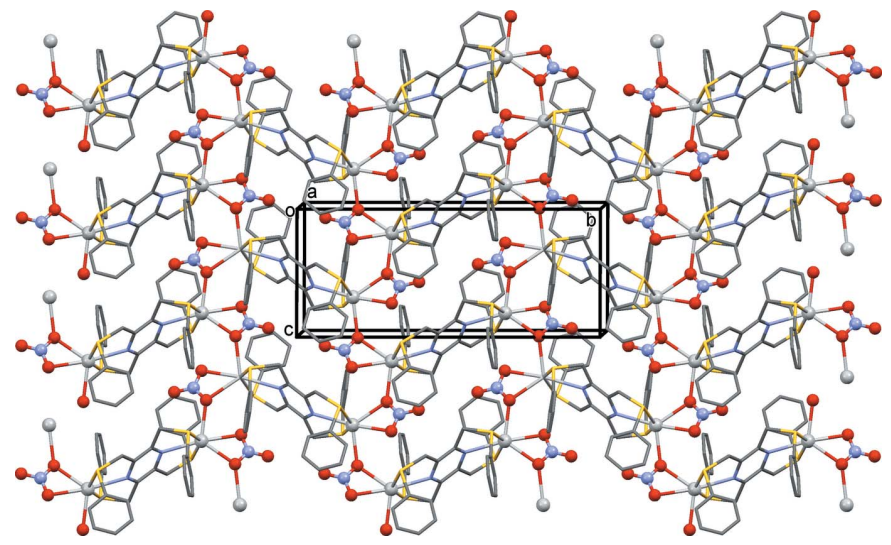

Figure 6

A view along the $a$ axis of compound (II), illustrating the role of the $\mathrm{NO}_{3}{ }^{-}$ anion in forming the network structure. $\mathrm{H}$ atoms have been omitted for clarity 


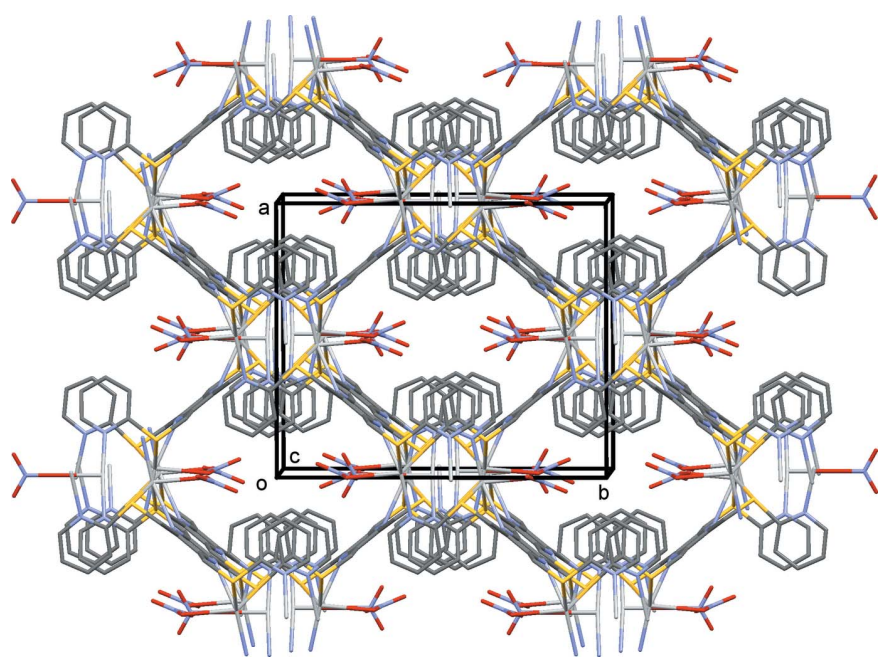

Figure 7

A view along the $c$ axis of compound (III). $\mathrm{H}$ atoms have been omitted for clarity

$\mathrm{N}$ (pyrazine), from 2.48 to $2.79 \AA$ for $\mathrm{Ag}-\mathrm{S}$, and 1.90 to $2.99 \AA$ for $\mathrm{Ag}-\mathrm{N}$ (pyridine).

\section{Supramolecular features}

In the crystal of (I), the metal-organic chains (Fig. 4) propagate along [101]. They are linked via a number of $\mathrm{C}-\mathrm{H} \cdots \mathrm{O}$ hydrogen bonds (Table 4), forming a three-dimensional supramolecular structure, as illustrated in Fig. 5.

In the crystal of (II), the metal-organic networks extend parallel to the $b c$ plane and stack up the $a$ axis (Fig. 6), but there are no significant intermolecular interactions present between the layers.

In the crystal of (III), the metal-organic framework (Fig. 7) is reinforced by a number of $\mathrm{C}-\mathrm{H} \cdots \mathrm{O}$ hydrogen bonds (Table 5). The voids in this three-dimensional structure, occupied by disordered solvent molecules, amount to only $\mathrm{ca}$ $3.7 \%$ of the total volume of the unit cell.

\section{Database survey}

A search of the Cambridge Structural Database (Version 5.38, first update November 2016; Groom et al., 2016) for tetrakissubstituted pyrazine ligands gave 774 hits, which include 194 hits for compounds involving tetramethylpyrazine. The first such ligand, tetrakis-2,3,5,6-(2'-pyridyl)pyrazine, was synthesized by Goodwin \& Lions (1959), and the crystal structures of three polymorphs have been reported; a monoclininc $P 2_{1} / n$ polymorph (VUKGAJ01; Bock et al., 1992), a tetragonal $I 4_{1} / a$ polymorph (VUKGAJ; Greaves \& Stoeckli-Evans, 1992) and a second monoclinic $C 2 / c$ polymorph (VUKGAJ03; Behrens \& Rehder, 2009). The most recent tetrakis-substituted pyrazine ligand to be described is $N, N^{\prime}, N^{\prime \prime}, N^{\prime \prime \prime}$-tetraethylpyrazine2,3,5,6-tetracarboxamide (OSUTIH; Lohrman et al., 2016). In the last update of the CSD there are a total of three tetrakis- substituted thioether pyrazine compounds, viz. two polymorphs of compound 2,3,5,6-tetrakis(naphthalen-2-ylsulfanylmethyl)pyrazine (Pacifico \& Stoeckli-Evans, 2004), and the ligands $\mathbf{L} \mathbf{1}$ and $\mathbf{L 2}$.

The role of the anion in coordination chemistry is often essential for the formation of multi-dimensional structures. The nitrate anion can be present as an isolated anion, coordinating to the metal atom or even bridging metal atoms. A search of the CSD for silver nitrate complexes yielded 2192 hits, among which it was noted that the nitrate anion can coordinate in at least 10 different manners. In the present study, three different situations are observed. In (I), the nitrate anion is present as an isolated anion. Its role here is to form $\mathrm{C}-\mathrm{H}$. . O hydrogen bonds, resulting in the formation of a three-dimensional supramolecular structure (Fig. 5 and Table 4). In (II), the nitrate anion is essential in forming the network structure. The -Ag-L2-Ag-L2- chains, which propagate along [010], are linked by the nitrate anion in the [001] direction, so forming the metal-organic network (Fig. 6 and Table 2). Finally, there are two independent nitrate anions present in (III). They coordinate to the metal atoms in different manners, but they do not appear to be the essential elements in forming the three-dimensional framework (Fig. 7 and Table 3). Here, it is the presence of the pyridine rings, which twist about the $\mathrm{S}-\mathrm{C}_{\mathrm{ar}}$ bonds, that enables the metal atoms to cross-link, so forming the metal-organic framework.

\section{Synthesis and crystallization}

\section{Compound (I):}

A solution of $\mathbf{L 1}(50 \mathrm{mg}, 0.16 \mathrm{mmol}$; Assoumatine \& Stoeckli-Evans, 2014a) in $\mathrm{CH}_{2} \mathrm{Cl}_{2}(5 \mathrm{ml})$ was introduced into a $16 \mathrm{~mm}$ diameter glass tube and layered with $\mathrm{MeCN}(2 \mathrm{ml})$ as a buffer zone. Then a solution of $\mathrm{AgNO}_{3}(27 \mathrm{mg}, 0.16 \mathrm{mmol})$ in $\mathrm{MeCN}(5 \mathrm{ml})$ was added very gently to avoid possible mixing. The glass tube was sealed and left in the dark at room temperature for at least two weeks, whereupon yellow platelike crystals of complex (I) were isolated at the interface between the two solutions. IR ( $\mathrm{KBr}$ disc, $\left.\mathrm{cm}^{-1}\right): v=2985 w$, $2912 w, 1406$ bm, 1341 bs, $1141 w, 1115 w, 982 w, 828 w, 777 w$, $701 v w, 478 v w$.

\section{Compound (II):}

A solution of $\mathbf{L 2}(50 \mathrm{mg}, 0.09 \mathrm{mmol}$; Assoumatine et al., $2007)$ in THF (5 ml) was introduced into a $16 \mathrm{~mm}$ diameter glass tube and layered with $\mathrm{MeCN}(2 \mathrm{ml})$ as a buffer zone. Then a solution of $\mathrm{AgNO}_{3}(15 \mathrm{mg}, 0.09 \mathrm{mmol})$ in $\mathrm{MeCN}$ $(5 \mathrm{ml})$ was added very gently to avoid possible mixing. The glass tube was sealed and left in the dark at room temperature for at least three weeks, whereupon yellow block-like crystals of complex (II) were isolated from the bottom of the tube. IR $\left(\mathrm{KBr}\right.$ disc, $\left.\mathrm{cm}^{-1}\right): v=3053 v w, 2962 v w, 2927 v w, 1583 w, 1480$ $w, 1386$ bs, $1278 v s, 1133 v w, 1023 w, 850 v w, 738 s, 690 m, 495$ $v w, 478 v w$.

\section{Compound (III):}

A solution of $\mathbf{L 3}$ (50 mg, $0.09 \mathrm{mmol}$; Assoumatine \& Stoeckli-Evans, 2016) in $\mathrm{CHCl}_{3}(5 \mathrm{ml})$ was introduced into a $16 \mathrm{~mm}$ diameter glass tube and layered with $\mathrm{MeCN}(2 \mathrm{ml})$ as a 
Table 6

Experimental details.

(I)

Crystal data

Chemical formula

$M_{\mathrm{r}}$

Crystal system, space group

Temperature (K)

$a, b, c(\AA)$

$\beta\left({ }^{\circ}\right)$
$V\left(\AA^{3}\right)$

$Z$

Radiation type

$\mu\left(\mathrm{mm}^{-1}\right)$

Crystal size (mm)

Data collection

Diffractometer

Absorption correction

$T_{\min }, T_{\max }$

No. of measured, independent and

observed $[I>2 \sigma(I)]$ reflections

$R_{\text {int }}$

$(\sin \theta / \lambda)_{\max }\left(\AA^{-1}\right)$

Refinement

$R\left[F^{2}>2 \sigma\left(F^{2}\right)\right], w R\left(F^{2}\right), S$

No. of reflections

No. of parameters

$\mathrm{H}$-atom treatment

$\Delta \rho_{\max }, \Delta \rho_{\min }\left(\mathrm{e} \AA^{-3}\right)$

\begin{tabular}{|c|c|}
\hline$\left[\mathrm{Ag}\left(\mathrm{C}_{12} \mathrm{H}_{20} \mathrm{~N}_{2} \mathrm{~S}_{4}\right]\left(\mathrm{NO}_{3}\right)\right.$ & {$\left[\mathrm{Ag}_{2}\left(\mathrm{NO}_{3}\right)_{2}\left(\mathrm{C}_{32} \mathrm{H}_{28} \mathrm{~N}_{2} \mathrm{~S}_{4}\right)\right]$} \\
\hline 490.42 & 908.56 \\
\hline Monoclinic, $P 2_{1} / n$ & Monoclinic, $P 2_{1} / c$ \\
\hline 293 & 293 \\
\hline $10.167(2), 13.482(3), 13.377$ (3) & $\begin{array}{l}11.8437(14), 18.5674(14) \\
\quad 7.8444(12)\end{array}$ \\
\hline $100.838(19)$ & $96.856(11)$ \\
\hline $1800.9(7)$ & 1712.7 (4) \\
\hline 4 & 2 \\
\hline Мо $K \alpha$ & Mo $K \alpha$ \\
\hline 1.60 & 1.44 \\
\hline $0.61 \times 0.61 \times 0.17$ & $0.46 \times 0.46 \times 0.38$ \\
\hline
\end{tabular}

(II)

$0.46 \times 0.46 \times 0.38$

Stoe AED2 4-circle
-
-
$3178,3178,2606$
0
0.606

$0.045,0.100,1.16$

3178

218

3318
207

$\mathrm{H}$-atom parameters constrained $1.97,-1.50$
(III)

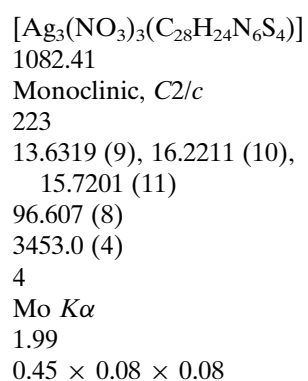

STOE IPDS1

Multi-scan (MULABS; Spek, 2009)

$0.949,1.000$

$13264,3311,1936$

0.072

0.614

$0.030,0.052,0.76$
3311
242
H-atom parameters constrained
$0.43,-0.44$

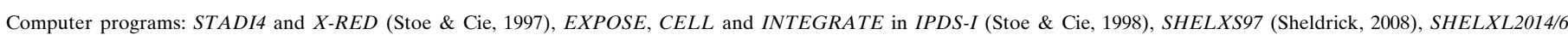
(Sheldrick, 2015), Mercury (Macrae et al., 2008), PLATON (Spek, 2009) and publCIF (Westrip, 2010).

buffer zone. Then a solution of $\mathrm{AgNO}_{3}(15 \mathrm{mg}, 0.09 \mathrm{mmol})$ in $\mathrm{MeCN}(5 \mathrm{ml})$ was added very gently to avoid possible mixing. The glass tube was sealed and left in the dark at room temperature for at least two weeks, whereupon pale-yellow needle-like crystals of complex (III) were isolated at the interface between the two solutions. IR ( $\mathrm{KBr}$ disc, $\left.\mathrm{cm}^{-1}\right): v=$ $3097 v w, 2899 v w, 1581 \mathrm{~m}, 1562 w, 1460 \mathrm{~m}, 1386 \mathrm{bs}, 1305 \mathrm{bs}$, $1163 w, 1126 w, 1032 v w, 1004 v w, 825 v w, 759 m, 723 v w$, $461 v w$.

\section{Refinement}

Crystal data, data collection and structure refinement details are summarized in Table 6. Complexes (I) and (II) were measured at $293 \mathrm{~K}$ on a four-circle diffractometer, while complex (III) was measured at $223 \mathrm{~K}$ on a one-circle imageplate diffractometer. In complex (I), the nitrate ion is positionally disordered and atoms $\mathrm{O} 12 A / \mathrm{O} 12 B$ and $\mathrm{O} 13 A / \mathrm{O} 13 B$ were refined with a fixed occupancy ratio of 0.5:0.5. No absorption correction was applied for complex (II) owing to the irregular shape of the crystal, and as there were no suitable reflections for $\psi$ scans. For complex (III), a region of disordered electron density (25 electrons for a solvent-accessible volume of $130 \AA^{3}$ ) was corrected for using the SQUEEZE routine in PLATON (Spek, 2015). Their formula mass and unit-cell characteristics were not taken into account for the final model. For complexes (I) and (II), only one equivalent of data were measured, hence $R_{\text {int }}=0$. In all three complexes, the $\mathrm{H}$ atoms were included in calculated positions and refined as riding: $\mathrm{C}-\mathrm{H}=0.96-0.97 \AA$ for (I), 0.93-0.97 $\AA$ for (II) and 0.94-0.98 $\AA$ for (III), with $U_{\text {iso }}(\mathrm{H})=1.5 U_{\text {eq }}(\mathrm{C}-$ methyl $)$ and $1.2 U_{\text {eq }}(\mathrm{C})$ for other $\mathrm{H}$ atoms.

\section{Funding information}

Funding for this research was provided by: Swiss National Science Foundation; University of Neuchâtel.

\section{References}

Addison, A. W., Rao, T. N., Reedijk, J., van Rijn, J. \& Verschoor, G. C. (1984). J. Chem. Soc. Dalton Trans. pp. 1349-1356.

Assoumatine, T. (1999). PhD thesis, University of Neuchâtel, Switzerland.

Assoumatine, T., Gasser, G. \& Stoeckli-Evans, H. (2007). Acta Cryst. C63, o219-o222.

Assoumatine, T. \& Stoeckli-Evans, H. (2014a). Acta Cryst. E70, 5153.

Assoumatine, T. \& Stoeckli-Evans, H. (2014b). Acta Cryst. E70, o887o888.

Assoumatine, T. \& Stoeckli-Evans, H. (2016). IUCrData, 1, x161977.

Behrens, A. \& Rehder, D. (2009). Private Communication (CCDC No. 261615, refcode VUKGAJ03). CCDC, Cambridge, England.

Black, C. A. \& Hanton, L. R. (2007). Cryst. Growth Des. 7, 1868-1871.

Bock, H., Vaupel, T., Näther, C., Ruppert, K. \& Havlas, Z. (1992). Angew. Chem. Int. Ed. Engl. 31, 299-301.

Caradoc-Davies, P. L. \& Hanton, L. R. (2001). Chem. Commun. pp. 1098-1099. 
Caradoc-Davies, P. L., Hanton, L. R. \& Henderson, W. (2001). J. Chem. Soc. Dalton Trans. pp. 2749-2755.

Goodwin, H. A. \& Lions, F. (1959). J. Am. Chem. Soc. 81, 6415-6422.

Greaves, B. \& Stoeckli-Evans, H. (1992). Acta Cryst. C48, 2269-2271.

Groom, C. R., Bruno, I. J., Lightfoot, M. P. \& Ward, S. C. (2016). Acta Cryst. B72, 171-179.

Lohrman, J., Telikepalli, H., Johnson, T. S., Jackson, T. A., Day, V. W. \& Bowman-James, K. (2016). Inorg. Chem. 55, 5098-5100.

Macrae, C. F., Bruno, I. J., Chisholm, J. A., Edgington, P. R., McCabe, P., Pidcock, E., Rodriguez-Monge, L., Taylor, R., van de Streek, J. \& Wood, P. A. (2008). J. Appl. Cryst. 41, 466-470.
Pacifico, J. \& Stoeckli-Evans, H. (2004). Acta Cryst. C60, o152-o155. Sheldrick, G. M. (2008). Acta Cryst. A64, 112-122.

Sheldrick, G. M. (2015). Acta Cryst. C71, 3-8.

Spek, A. L. (2009). Acta Cryst. D65, 148-155.

Spek, A. L. (2015). Acta Cryst. C71, 9-18.

Stoe \& Cie (1997). STADI4 and X-RED. Stoe \& Cie GmbH, Darmstadt, Germany.

Stoe \& Cie (1998). IPDS-I Bedienungshandbuch. Stoe \& Cie GmbH, Darmstadt, Germany.

Westrip, S. P. (2010). J. Appl. Cryst. 43, 920-925. 


\section{supporting information}

Acta Cryst. (2017). E73, 434-440 [https://doi.org/10.1107/S2056989017002791]

Silver(I) nitrate complexes of three tetrakis-thioether-substituted pyrazine ligands: metal-organic chain, network and framework structures

\section{Tokouré Assoumatine and Helen Stoeckli-Evans}

Computing details

Data collection: STADI4 (Stoe \& Cie, 1997) for (I), (II); EXPOSE in IPDS-I (Stoe \& Cie, 1998) for (III). Cell refinement: STADI4 (Stoe \& Cie, 1997) for (I), (II); CELL in IPDS-I (Stoe \& Cie, 1998) for (III). Data reduction: X-RED (Stoe \& Cie, 1997) for (I), (II); INTEGRATE in IPDS-I (Stoe \& Cie, 1998) for (III). For all compounds, program(s) used to solve structure: SHELXS97 (Sheldrick, 2008); program(s) used to refine structure: SHELXL2014/6 (Sheldrick, 2015); molecular graphics: Mercury (Macrae et al., 2008); software used to prepare material for publication: SHELXL2014/6 (Sheldrick, 2015), PLATON (Spek, 2009) and publCIF (Westrip, 2010).

(I) catena-Poly[[silver(I)- $\mu$-2,3,5,6-tetrakis[(methylsulfanyl)methyl]pyrazine] nitrate]

Crystal data

$\left[\mathrm{Ag}\left(\mathrm{C}_{12} \mathrm{H}_{20} \mathrm{~N}_{2} \mathrm{~S}_{4}\right]\left(\mathrm{NO}_{3}\right)\right.$

$M_{r}=490.42$

Monoclinic, $P 2_{1} / n$

$a=10.167(2) \AA$

$b=13.482(3) \AA$

$c=13.377(3) \AA$

$\beta=100.838(19)^{\circ}$

$V=1800.9(7) \AA^{3}$

$Z=4$

Data collection

Stoe AED2 4-circle diffractometer

Radiation source: fine-focus sealed tube Graphite monochromator $\omega / 2 \theta$ scans

Absorption correction: analytical (ABST; Spek, 2009)

$T_{\min }=0.457, T_{\max }=0.789$

3318 measured reflections

Refinement

Refinement on $F^{2}$

Least-squares matrix: full

$R\left[F^{2}>2 \sigma\left(F^{2}\right)\right]=0.056$

$w R\left(F^{2}\right)=0.161$

$S=1.09$

3318 reflections
$F(000)=992$

$D_{\mathrm{x}}=1.809 \mathrm{Mg} \mathrm{m}^{-3}$

Mo $K \alpha$ radiation, $\lambda=0.71073 \AA$

Cell parameters from 31 reflections

$\theta=14.1-19.7^{\circ}$

$\mu=1.60 \mathrm{~mm}^{-1}$

$T=293 \mathrm{~K}$

Plate, yellow

$0.61 \times 0.61 \times 0.17 \mathrm{~mm}$

3318 independent reflections

2857 reflections with $I>2 \sigma(I)$

$R_{\text {int }}=0.000$

$\theta_{\max }=25.6^{\circ}, \theta_{\min }=2.2^{\circ}$

$h=-11 \rightarrow 12$

$k=0 \rightarrow 16$

$l=0 \rightarrow 16$

2 standard reflections every $120 \mathrm{~min}$ intensity decay: $5 \%$

207 parameters

0 restraints

Primary atom site location: structure-invariant direct methods

Secondary atom site location: difference Fourier map 
Hydrogen site location: inferred from neighbouring sites

$\mathrm{H}$-atom parameters constrained

$w=1 /\left[\sigma^{2}\left(F_{\mathrm{o}}^{2}\right)+(0.0974 P)^{2}+4.9525 P\right]$

where $P=\left(F_{\mathrm{o}}^{2}+2 F_{\mathrm{c}}^{2}\right) / 3$

$(\Delta / \sigma)_{\max }<0.001$

\author{
$\Delta \rho_{\max }=1.97$ e $\AA^{-3}$ \\ $\Delta \rho_{\min }=-1.50$ e $\AA^{-3}$ \\ Extinction correction: SHELXL-2016/6 \\ (Sheldrick 2015), \\ $\mathrm{Fc}^{*}=\mathrm{kFc}\left[1+0.001 \times \mathrm{Fc}^{2} \lambda^{3} / \sin (2 \theta)\right]^{-1 / 4}$ \\ Extinction coefficient: 0.0035 (8)
}

\section{Special details}

Geometry. All esds (except the esd in the dihedral angle between two 1.s. planes) are estimated using the full covariance matrix. The cell esds are taken into account individually in the estimation of esds in distances, angles and torsion angles; correlations between esds in cell parameters are only used when they are defined by crystal symmetry. An approximate (isotropic) treatment of cell esds is used for estimating esds involving 1.s. planes.

Fractional atomic coordinates and isotropic or equivalent isotropic displacement parameters $\left(\AA^{2}\right)$

\begin{tabular}{|c|c|c|c|c|c|}
\hline & $x$ & $y$ & $z$ & $U_{\text {iso }} * / U_{\text {eq }}$ & Occ. $(<1)$ \\
\hline Ag1 & $0.23796(5)$ & $0.48987(4)$ & $0.75616(3)$ & $0.0401(2)$ & \\
\hline $\mathrm{S} 1$ & $0.43682(14)$ & $0.37806(10)$ & $0.73224(10)$ & $0.0323(3)$ & \\
\hline $\mathrm{S} 2$ & $0.11067(14)$ & $0.50053(10)$ & $0.90707(11)$ & $0.0320(3)$ & \\
\hline $\mathrm{S} 3$ & $-0.06264(16)$ & $0.24651(10)$ & $0.63652(12)$ & $0.0413(4)$ & \\
\hline S4 & $-0.26369(13)$ & $0.33208(10)$ & $0.31050(10)$ & $0.0330(3)$ & \\
\hline N1 & $0.4152(4)$ & $0.4454(3)$ & $0.9286(3)$ & $0.0292(9)$ & \\
\hline N2 & $0.0779(5)$ & $0.4916(3)$ & $0.5959(4)$ & $0.0282(10)$ & \\
\hline $\mathrm{C} 1$ & $0.5407(5)$ & $0.4649(4)$ & $0.9166(4)$ & $0.0272(11)$ & \\
\hline $\mathrm{C} 2$ & $0.5796(6)$ & $0.4261(5)$ & $0.8198(4)$ & $0.0360(13)$ & \\
\hline $\mathrm{H} 2 \mathrm{~A}$ & 0.645719 & 0.373887 & 0.836842 & $0.043^{*}$ & \\
\hline $\mathrm{H} 2 \mathrm{~B}$ & 0.620036 & 0.479211 & 0.787138 & $0.043^{*}$ & \\
\hline $\mathrm{C} 3$ & $0.4998(7)$ & $0.3836(5)$ & $0.6154(5)$ & $0.0486(16)$ & \\
\hline $\mathrm{H} 3 \mathrm{~A}$ & 0.430481 & 0.364140 & 0.559808 & $0.073 *$ & \\
\hline $\mathrm{H} 3 \mathrm{~B}$ & 0.574665 & 0.339421 & 0.619518 & $0.073^{*}$ & \\
\hline $\mathrm{H} 3 \mathrm{C}$ & 0.527752 & 0.450091 & 0.604654 & $0.073^{*}$ & \\
\hline $\mathrm{C} 4$ & $0.3736(5)$ & $0.4814(4)$ & $1.0107(4)$ & $0.0268(11)$ & \\
\hline $\mathrm{C} 5$ & $0.2294(5)$ & $0.4566(4)$ & $1.0169(4)$ & $0.0322(11)$ & \\
\hline $\mathrm{H} 5 \mathrm{~A}$ & 0.207925 & 0.486153 & 1.078010 & $0.039 *$ & \\
\hline H5B & 0.220455 & 0.385218 & 1.022356 & $0.039 *$ & \\
\hline C6 & $0.1099(7)$ & $0.6312(5)$ & $0.9332(6)$ & $0.0512(16)$ & \\
\hline H6A & 0.047093 & 0.663787 & 0.880930 & $0.077^{*}$ & \\
\hline H6B & 0.197800 & 0.657895 & 0.934761 & $0.077^{*}$ & \\
\hline $\mathrm{H} 6 \mathrm{C}$ & 0.084358 & 0.641823 & 0.997925 & $0.077^{*}$ & \\
\hline $\mathrm{C} 7$ & $0.0317(5)$ & $0.4104(4)$ & $0.5426(4)$ & $0.0273(10)$ & \\
\hline $\mathrm{C} 10$ & $-0.0478(5)$ & $0.4186(4)$ & 0.4465 & $0.0265(10)$ & \\
\hline $\mathrm{C} 11$ & $-0.0956(5)$ & $0.3274(4)$ & $0.3842(4)$ & $0.0334(12)$ & \\
\hline H11A & -0.089767 & 0.271140 & 0.430044 & $0.040 *$ & \\
\hline H11B & -0.034496 & 0.315052 & 0.337994 & $0.040 *$ & \\
\hline $\mathrm{C} 8$ & $0.0713(5)$ & $0.3120(4)$ & $0.5925(4)$ & $0.0313(11)$ & \\
\hline H8A & 0.143563 & 0.322787 & 0.650060 & $0.038^{*}$ & \\
\hline H8B & 0.105664 & 0.270039 & 0.544339 & $0.038 *$ & \\
\hline $\mathrm{C} 9$ & $-0.0972(9)$ & $0.3316(5)$ & $0.7311(6)$ & $0.062(2)$ & \\
\hline H9A & -0.161874 & 0.302866 & 0.766452 & $0.093 *$ & \\
\hline
\end{tabular}




\begin{tabular}{|c|c|c|c|c|c|}
\hline H9B & -0.132267 & 0.392150 & 0.698934 & $0.093^{*}$ & \\
\hline $\mathrm{H} 9 \mathrm{C}$ & -0.016152 & 0.345284 & 0.778694 & $0.093^{*}$ & \\
\hline $\mathrm{C} 12$ & $-0.3592(7)$ & $0.3497(6)$ & $0.4101(6)$ & $0.0569(18)$ & \\
\hline $\mathrm{H} 12 \mathrm{~A}$ & -0.451820 & 0.359680 & 0.380318 & $0.085^{*}$ & \\
\hline H12B & -0.326070 & 0.406797 & 0.449937 & $0.085^{*}$ & \\
\hline $\mathrm{H} 12 \mathrm{C}$ & -0.350633 & 0.292137 & 0.453042 & $0.085^{*}$ & \\
\hline N10 & $0.2281(6)$ & $0.3702(5)$ & $0.3423(5)$ & $0.0544(15)$ & \\
\hline O11 & $0.2331(7)$ & $0.2979(5)$ & $0.3953(5)$ & $0.0842(13)$ & \\
\hline $\mathrm{O} 12 \mathrm{~A}$ & $0.2834(16)$ & $0.4447(11)$ & $0.3565(11)$ & $0.0842(13)$ & 0.5 \\
\hline O13A & $0.1631(17)$ & $0.3451(10)$ & $0.2526(12)$ & $0.0842(13)$ & 0.5 \\
\hline O12B & $0.2588(16)$ & $0.4507(11)$ & $0.4035(11)$ & $0.0842(13)$ & 0.5 \\
\hline O13B & $0.1847(18)$ & $0.3871(10)$ & $0.2537(12)$ & $0.0842(13)$ & 0.5 \\
\hline
\end{tabular}

Atomic displacement parameters $\left(\AA^{2}\right)$

\begin{tabular}{|c|c|c|c|c|c|c|}
\hline & $U^{11}$ & $U^{22}$ & $U^{33}$ & $U^{12}$ & $U^{13}$ & $U^{23}$ \\
\hline Ag1 & $0.0397(3)$ & $0.0501(3)$ & 0.0284 & 0.00285 (18) & $0.0014(2)$ & 0.00409 (17) \\
\hline S1 & $0.0349(7)$ & $0.0359(7)$ & $0.0280(7)$ & $-0.0023(5)$ & $0.0105(5)$ & $-0.0025(5)$ \\
\hline S2 & $0.0243(7)$ & $0.0424(8)$ & $0.0293(7)$ & $-0.0031(5)$ & $0.0056(5)$ & $-0.0003(5)$ \\
\hline S3 & $0.0473(9)$ & $0.0327(7)$ & $0.0422(8)$ & $-0.0100(6)$ & $0.0044(7)$ & $0.0024(6)$ \\
\hline S4 & $0.0312(7)$ & $0.0361(7)$ & $0.0315(7)$ & $-0.0046(5)$ & $0.0056(5)$ & -0.0069 \\
\hline N1 & $0.022(2)$ & $0.040(2)$ & $0.027(2)$ & $-0.0010(18)$ & $0.0078(18)$ & $-0.0002(18)$ \\
\hline N2 & $0.030(2)$ & $0.029(2)$ & $0.027(2)$ & $0.0020(17)$ & $0.0065(19)$ & $-0.0017(16)$ \\
\hline $\mathrm{C} 1$ & $0.028(3)$ & $0.035(3)$ & $0.020(2)$ & $0.005(2)$ & $0.007(2)$ & $0.0033(19)$ \\
\hline $\mathrm{C} 2$ & 0.029 & $0.054(3)$ & $0.029(3)$ & $0.002(2)$ & $0.016(2)$ & $-0.006(2)$ \\
\hline $\mathrm{C} 3$ & $0.061(4)$ & $0.056(4)$ & $0.031(3)$ & -0.010 & $0.017(3)$ & $-0.011(3)$ \\
\hline $\mathrm{C} 4$ & $0.022(3)$ & $0.037(3)$ & $0.023(2)$ & $0.005(2)$ & $0.007(2)$ & $0.004(2)$ \\
\hline $\mathrm{C} 5$ & $0.023(3)$ & $0.041(3)$ & $0.034(3)$ & $-0.005(2)$ & $0.010(2)$ & $0.005(2)$ \\
\hline C6 & $0.060(4)$ & $0.038(3)$ & $0.059(4)$ & 0.003 & $0.022(3)$ & $0.004(3)$ \\
\hline $\mathrm{C} 7$ & $0.025(2)$ & $0.028(2)$ & $0.030(3)$ & $-0.0005(19)$ & $0.008(2)$ & $-0.002(2)$ \\
\hline $\mathrm{C} 10$ & $0.024(2)$ & $0.028(2)$ & $0.028(2)$ & $0.0012(19)$ & $0.007(2)$ & $-0.002(2)$ \\
\hline C11 & $0.031(3)$ & $0.033(3)$ & $0.034(3)$ & $0.000(2)$ & $0.001(2)$ & -0.009 (2) \\
\hline $\mathrm{C} 8$ & $0.034(3)$ & 0.031 & $0.027(3)$ & $0.001(2)$ & $0.000(2)$ & $0.003(2)$ \\
\hline C9 & $0.077(5)$ & $0.046(4)$ & $0.076(5)$ & -0.009 & $0.047(4)$ & $-0.005(4)$ \\
\hline $\mathrm{C} 12$ & $0.056(4)$ & $0.062(4)$ & $0.063(4)$ & $-0.006(3)$ & $0.036(4)$ & $-0.003(3)$ \\
\hline N10 & $0.038(3)$ & $0.061(4)$ & $0.068(4)$ & $-0.006(3)$ & 0.020 & $0.006(3)$ \\
\hline O11 & $0.109(4)$ & 0.071 & $0.076(3)$ & $-0.016(3)$ & $0.026(3)$ & $0.011(3)$ \\
\hline $\mathrm{O} 12 \mathrm{~A}$ & 0.109 (4) & 0.071 & $0.076(3)$ & $-0.016(3)$ & $0.026(3)$ & $0.011(3)$ \\
\hline O13A & $0.109(4)$ & 0.071 & $0.076(3)$ & $-0.016(3)$ & $0.026(3)$ & $0.011(3)$ \\
\hline O12B & 0.109 (4) & $0.071(3)$ & $0.076(3)$ & $-0.016(3)$ & $0.026(3)$ & $0.011(3)$ \\
\hline O13B & 0.109 (4) & 0.071 & $0.076(3)$ & $-0.016(3)$ & $0.026(3)$ & $0.011(3)$ \\
\hline
\end{tabular}

Geometric parameters $\left(\AA,{ }^{\circ}\right)$

\begin{tabular}{llll}
\hline $\mathrm{Ag} 1-\mathrm{N} 1$ & $2.714(4)$ & $\mathrm{C} 4-\mathrm{C} 5$ & $1.522(7)$ \\
$\mathrm{Ag} 1-\mathrm{N} 2$ & $2.436(5)$ & $\mathrm{C} 5-\mathrm{H} 5 \mathrm{~A}$ & 0.9700 \\
$\mathrm{Ag} 1-\mathrm{S} 1$ & $2.5895(15)$ & $\mathrm{C} 5-\mathrm{H} 5 \mathrm{~B}$ & 0.9700 \\
$\mathrm{Ag} 1-\mathrm{S} 2$ & $2.5987(16)$ & $\mathrm{C} 6-\mathrm{H} 6 \mathrm{~A}$ & 0.9600
\end{tabular}




\begin{tabular}{|c|c|c|c|}
\hline $\mathrm{Ag} 1-\mathrm{S} 4^{\mathrm{i}}$ & $2.5910(15)$ & C6-H6B & 0.9600 \\
\hline $\mathrm{S} 1-\mathrm{C} 3$ & $1.798(6)$ & $\mathrm{C} 6-\mathrm{H} 6 \mathrm{C}$ & 0.9600 \\
\hline $\mathrm{S} 1-\mathrm{C} 2$ & $1.805(6)$ & $\mathrm{C} 7-\mathrm{C} 10$ & $1.389(7)$ \\
\hline $\mathrm{S} 2-\mathrm{C} 6$ & $1.797(7)$ & $\mathrm{C} 7-\mathrm{C} 8$ & $1.506(7)$ \\
\hline $\mathrm{S} 2-\mathrm{C} 5$ & $1.817(6)$ & $\mathrm{C} 10-\mathrm{C} 11$ & $1.513(7)$ \\
\hline $\mathrm{S} 3-\mathrm{C} 9$ & $1.791(7)$ & $\mathrm{C} 11-\mathrm{H} 11 \mathrm{~A}$ & 0.9700 \\
\hline $\mathrm{S} 3-\mathrm{C} 8$ & $1.812(6)$ & $\mathrm{C} 11-\mathrm{H} 11 \mathrm{~B}$ & 0.9700 \\
\hline $\mathrm{S} 4-\mathrm{C} 11$ & $1.806(5)$ & $\mathrm{C} 8-\mathrm{H} 8 \mathrm{~A}$ & 0.9700 \\
\hline $\mathrm{S} 4-\mathrm{C} 12$ & $1.806(7)$ & $\mathrm{C} 8-\mathrm{H} 8 \mathrm{~B}$ & 0.9700 \\
\hline $\mathrm{N} 1-\mathrm{C} 4$ & $1.341(7)$ & C9-H9A & 0.9600 \\
\hline $\mathrm{N} 1-\mathrm{C} 1$ & $1.342(7)$ & C9-H9B & 0.9600 \\
\hline $\mathrm{N} 2-\mathrm{C} 7$ & $1.342(7)$ & $\mathrm{C} 9-\mathrm{H} 9 \mathrm{C}$ & 0.9600 \\
\hline $\mathrm{N} 2-\mathrm{C} 10^{\mathrm{i}}$ & $1.348(6)$ & $\mathrm{C} 12-\mathrm{H} 12 \mathrm{~A}$ & 0.9600 \\
\hline $\mathrm{C} 1-\mathrm{C} 4^{\mathrm{ii}}$ & $1.381(8)$ & $\mathrm{C} 12-\mathrm{H} 12 \mathrm{~B}$ & 0.9600 \\
\hline $\mathrm{C} 1-\mathrm{C} 2$ & $1.517(7)$ & $\mathrm{C} 12-\mathrm{H} 12 \mathrm{C}$ & 0.9600 \\
\hline $\mathrm{C} 2-\mathrm{H} 2 \mathrm{~A}$ & 0.9700 & $\mathrm{~N} 10-\mathrm{O} 12 \mathrm{~A}$ & $1.148(15)$ \\
\hline $\mathrm{C} 2-\mathrm{H} 2 \mathrm{~B}$ & 0.9700 & $\mathrm{~N} 10-\mathrm{O} 11$ & $1.202(8)$ \\
\hline $\mathrm{C} 3-\mathrm{H} 3 \mathrm{~A}$ & 0.9600 & $\mathrm{~N} 10-\mathrm{O} 13 \mathrm{~B}$ & $1.205(17)$ \\
\hline C $3-\mathrm{H} 3 \mathrm{~B}$ & 0.9600 & $\mathrm{~N} 10-\mathrm{O} 13 \mathrm{~A}$ & $1.301(17)$ \\
\hline $\mathrm{C} 3-\mathrm{H} 3 \mathrm{C}$ & 0.9600 & $\mathrm{~N} 10-\mathrm{O} 12 \mathrm{~B}$ & $1.360(16)$ \\
\hline $\mathrm{N} 1-\mathrm{Ag} 1-\mathrm{N} 2$ & $167.75(13)$ & $\mathrm{C} 4-\mathrm{C} 5-\mathrm{H} 5 \mathrm{~B}$ & 109.1 \\
\hline $\mathrm{N} 1-\mathrm{Ag} 1-\mathrm{S} 1$ & $64.36(9)$ & $\mathrm{S} 2-\mathrm{C} 5-\mathrm{H} 5 \mathrm{~B}$ & 109.1 \\
\hline $\mathrm{N} 1-\mathrm{Ag} 1-\mathrm{S} 2$ & $72.54(9)$ & $\mathrm{H} 5 \mathrm{~A}-\mathrm{C} 5-\mathrm{H} 5 \mathrm{~B}$ & 107.8 \\
\hline $\mathrm{N} 1-\mathrm{Ag} 1-\mathrm{S} 4^{\mathrm{i}}$ & $113.79(9)$ & $\mathrm{S} 2-\mathrm{C} 6-\mathrm{H} 6 \mathrm{~A}$ & 109.5 \\
\hline $\mathrm{N} 2-\mathrm{Ag} 1-\mathrm{S} 1$ & $107.74(11)$ & $\mathrm{S} 2-\mathrm{C} 6-\mathrm{H} 6 \mathrm{~B}$ & 109.5 \\
\hline $\mathrm{N} 2-\mathrm{Ag} 1-\mathrm{S} 2$ & $109.60(11)$ & $\mathrm{H} 6 \mathrm{~A}-\mathrm{C} 6-\mathrm{H} 6 \mathrm{~B}$ & 109.5 \\
\hline $\mathrm{N} 2-\mathrm{Ag} 1-\mathrm{S} 4^{\mathrm{i}}$ & $77.43(10)$ & $\mathrm{S} 2-\mathrm{C} 6-\mathrm{H} 6 \mathrm{C}$ & 109.5 \\
\hline $\mathrm{S} 1-\mathrm{Ag} 1-\mathrm{S} 2$ & $129.99(5)$ & $\mathrm{H} 6 \mathrm{~A}-\mathrm{C} 6-\mathrm{H} 6 \mathrm{C}$ & 109.5 \\
\hline $\mathrm{S} 1-\mathrm{Ag} 1-\mathrm{S} 4^{\mathrm{i}}$ & $111.41(5)$ & $\mathrm{H} 6 \mathrm{~B}-\mathrm{C} 6-\mathrm{H} 6 \mathrm{C}$ & 109.5 \\
\hline $\mathrm{S} 4 \mathrm{i}-\mathrm{Ag} 1-\mathrm{S} 2$ & $108.26(5)$ & $\mathrm{N} 2-\mathrm{C} 7-\mathrm{C} 10$ & $120.8(5)$ \\
\hline $\mathrm{C} 3-\mathrm{S} 1-\mathrm{C} 2$ & $100.1(3)$ & $\mathrm{N} 2-\mathrm{C} 7-\mathrm{C} 8$ & $116.5(4)$ \\
\hline $\mathrm{C} 3-\mathrm{S} 1-\mathrm{Ag} 1$ & $119.8(2)$ & $\mathrm{C} 10-\mathrm{C} 7-\mathrm{C} 8$ & $122.8(4)$ \\
\hline $\mathrm{C} 2-\mathrm{S} 1-\mathrm{Ag} 1$ & $105.22(19)$ & $\mathrm{N} 2{ }^{\mathrm{i}}-\mathrm{C} 10-\mathrm{C} 7$ & $120.5(4)$ \\
\hline $\mathrm{C} 6-\mathrm{S} 2-\mathrm{C} 5$ & $100.9(3)$ & $\mathrm{N} 22^{\mathrm{i}}-\mathrm{C} 10-\mathrm{C} 11$ & $118.3(5)$ \\
\hline $\mathrm{C} 6-\mathrm{S} 2-\mathrm{Ag} 1$ & $103.1(2)$ & $\mathrm{C} 7-\mathrm{C} 10-\mathrm{C} 11$ & $121.1(4)$ \\
\hline $\mathrm{C} 5-\mathrm{S} 2-\mathrm{Ag} 1$ & $104.98(18)$ & $\mathrm{C} 10-\mathrm{C} 11-\mathrm{S} 4$ & $116.4(4)$ \\
\hline $\mathrm{C} 9-\mathrm{S} 3-\mathrm{C} 8$ & $100.2(3)$ & $\mathrm{C} 10-\mathrm{C} 11-\mathrm{H} 11 \mathrm{~A}$ & 108.2 \\
\hline $\mathrm{C} 11-\mathrm{S} 4-\mathrm{C} 12$ & $100.8(3)$ & $\mathrm{S} 4-\mathrm{C} 11-\mathrm{H} 11 \mathrm{~A}$ & 108.2 \\
\hline $\mathrm{C} 11-\mathrm{S} 4-\mathrm{Ag} 1^{\mathrm{i}}$ & $94.24(19)$ & $\mathrm{C} 10-\mathrm{C} 11-\mathrm{H} 11 \mathrm{~B}$ & 108.2 \\
\hline $\mathrm{C} 12-\mathrm{S} 4-\mathrm{Ag} 1^{\mathrm{i}}$ & $103.6(3)$ & $\mathrm{S} 4-\mathrm{C} 11-\mathrm{H} 11 \mathrm{~B}$ & 108.2 \\
\hline $\mathrm{C} 4-\mathrm{N} 1-\mathrm{C} 1$ & $118.7(5)$ & $\mathrm{H} 11 \mathrm{~A}-\mathrm{C} 11-\mathrm{H} 11 \mathrm{~B}$ & 107.3 \\
\hline $\mathrm{C} 7-\mathrm{N} 2-\mathrm{C} 10^{\mathrm{i}}$ & $118.7(5)$ & $\mathrm{C} 7-\mathrm{C} 8-\mathrm{S} 3$ & $114.8(4)$ \\
\hline $\mathrm{C} 7-\mathrm{N} 2-\mathrm{Ag} 1$ & $124.7(3)$ & $\mathrm{C} 7-\mathrm{C} 8-\mathrm{H} 8 \mathrm{~A}$ & 108.6 \\
\hline $\mathrm{C} 10^{\mathrm{i}}-\mathrm{N} 2-\mathrm{Ag} 1$ & $116.1(3)$ & $\mathrm{S} 3-\mathrm{C} 8-\mathrm{H} 8 \mathrm{~A}$ & 108.6 \\
\hline $\mathrm{N} 1-\mathrm{C} 1-\mathrm{C} 4^{\mathrm{ii}}$ & $120.5(5)$ & $\mathrm{C} 7-\mathrm{C} 8-\mathrm{H} 8 \mathrm{~B}$ & 108.6 \\
\hline $\mathrm{N} 1-\mathrm{C} 1-\mathrm{C} 2$ & $116.1(5)$ & $\mathrm{S} 3-\mathrm{C} 8-\mathrm{H} 8 \mathrm{~B}$ & 108.6 \\
\hline $\mathrm{C} 44^{\mathrm{ii}}-\mathrm{C} 1-\mathrm{C} 2$ & $123.4(5)$ & $\mathrm{H} 8 \mathrm{~A}-\mathrm{C} 8-\mathrm{H} 8 \mathrm{~B}$ & 107.6 \\
\hline
\end{tabular}




$\begin{array}{ll}\mathrm{C} 1-\mathrm{C} 2-\mathrm{S} 1 & 111.8(4) \\ \mathrm{C} 1-\mathrm{C} 2-\mathrm{H} 2 \mathrm{~A} & 109.3 \\ \mathrm{~S} 1-\mathrm{C} 2-\mathrm{H} 2 \mathrm{~A} & 109.3 \\ \mathrm{C} 1-\mathrm{C} 2-\mathrm{H} 2 \mathrm{~B} & 109.3 \\ \mathrm{~S} 1-\mathrm{C} 2-\mathrm{H} 2 \mathrm{~B} & 109.3 \\ \mathrm{H} 2 \mathrm{~A}-\mathrm{C} 2-\mathrm{H} 2 \mathrm{~B} & 107.9 \\ \mathrm{~S} 1-\mathrm{C} 3-\mathrm{H} 3 \mathrm{~A} & 109.5 \\ \mathrm{~S} 1-\mathrm{C} 3-\mathrm{H} 3 \mathrm{~B} & 109.5 \\ \mathrm{H} 3 \mathrm{~A}-\mathrm{C} 3-\mathrm{H} 3 \mathrm{~B} & 109.5 \\ \mathrm{~S} 1-\mathrm{C} 3-\mathrm{H} 3 \mathrm{C} & 109.5 \\ \mathrm{H} 3 \mathrm{~A}-\mathrm{C} 3-\mathrm{H} 3 \mathrm{C} & 109.5 \\ \mathrm{H} 3 \mathrm{~B}-\mathrm{C} 3-\mathrm{H} 3 \mathrm{C} & 109.5 \\ \mathrm{~N} 1-\mathrm{C} 4-\mathrm{C} 1 \mathrm{ii} & 120.8(5) \\ \mathrm{N} 1-\mathrm{C} 4-\mathrm{C} 5 & 114.9(5) \\ \mathrm{C} 1{ }^{\mathrm{ii}-\mathrm{C} 4-\mathrm{C} 5} & 124.3(5) \\ \mathrm{C} 4-\mathrm{C} 5-\mathrm{S} 2 & 112.6(4) \\ \mathrm{C} 4-\mathrm{C} 5-\mathrm{H} 5 \mathrm{~A} & 109.1 \\ \mathrm{~S} 2-\mathrm{C} 5-\mathrm{H} 5 \mathrm{~A} & 109.1 \\ & \\ \mathrm{C} 4-\mathrm{N} 1-\mathrm{C} 1-\mathrm{C}{ }^{\mathrm{ii}} & 1.9(8) \\ \mathrm{C} 4-\mathrm{N} 1-\mathrm{C} 1-\mathrm{C} 2 & -177.3(5) \\ \mathrm{N} 1-\mathrm{C} 1-\mathrm{C} 2-\mathrm{S} 1 & 9.1(6) \\ \mathrm{C} 44^{\mathrm{ii}} \mathrm{C} 1-\mathrm{C} 2-\mathrm{S} 1 & -170.1(4) \\ \mathrm{C} 3-\mathrm{S} 1-\mathrm{C} 2-\mathrm{C} 1 & 159.3(4) \\ \mathrm{Ag} 1-\mathrm{S} 1-\mathrm{C} 2-\mathrm{C} 1 & 34.5(4) \\ \mathrm{C} 1-\mathrm{N} 1-\mathrm{C} 4-\mathrm{C} 1^{\mathrm{ii}} & -1.9(8) \\ \mathrm{C} 1-\mathrm{N} 1-\mathrm{C} 4-\mathrm{C} 5 & 179.2(5) \\ \mathrm{N} 1-\mathrm{C} 4-\mathrm{C} 5-\mathrm{S} 2 & -58.1(6) \\ \mathrm{C} 1{ }^{1 i}-\mathrm{C} 4-\mathrm{C} 5-\mathrm{S} 2 & 123.1(5) \\ \mathrm{C} 6-\mathrm{S} 2-\mathrm{C} 5-\mathrm{C} 4 & -74.8(5) \\ \mathrm{Ag} 1-\mathrm{S} 2-\mathrm{C} 5-\mathrm{C} 4 & 32.1(4) \\ \mathrm{C} 10-\mathrm{N} 2-\mathrm{C} 7-\mathrm{C} 10 & -1.3(8) \\ \mathrm{Ag} 1-\mathrm{N} 2-\mathrm{C} 7-\mathrm{C} 10 & -172.8(4) \\ & \end{array}$

$\mathrm{S} 3-\mathrm{C} 9-\mathrm{H} 9 \mathrm{~A}$

$\mathrm{S} 3-\mathrm{C} 9-\mathrm{H} 9 \mathrm{~B}$

$\mathrm{H} 9 \mathrm{~A}-\mathrm{C} 9-\mathrm{H} 9 \mathrm{~B}$

$\mathrm{S} 3-\mathrm{C} 9-\mathrm{H} 9 \mathrm{C}$

$\mathrm{H} 9 \mathrm{~A}-\mathrm{C} 9-\mathrm{H} 9 \mathrm{C}$

$\mathrm{H} 9 \mathrm{~B}-\mathrm{C} 9-\mathrm{H} 9 \mathrm{C}$

$\mathrm{S} 4-\mathrm{C} 12-\mathrm{H} 12 \mathrm{~A}$

$\mathrm{S} 4-\mathrm{C} 12-\mathrm{H} 12 \mathrm{~B}$

$\mathrm{H} 12 \mathrm{~A}-\mathrm{C} 12-\mathrm{H} 12 \mathrm{~B}$

$\mathrm{S} 4-\mathrm{C} 12-\mathrm{H} 12 \mathrm{C}$

$\mathrm{H} 12 \mathrm{~A}-\mathrm{C} 12-\mathrm{H} 12 \mathrm{C}$

$\mathrm{H} 12 \mathrm{~B}-\mathrm{C} 12-\mathrm{H} 12 \mathrm{C}$

$\mathrm{O} 12 \mathrm{~A}-\mathrm{N} 10-\mathrm{O} 11$

O11-N10-O13B

$\mathrm{O} 12 \mathrm{~A}-\mathrm{N} 10-\mathrm{O} 13 \mathrm{~A}$

O11-N10-O13A

O11-N10-O12B

$\mathrm{O} 13 \mathrm{~B}-\mathrm{N} 10-\mathrm{O} 12 \mathrm{~B}$

$\mathrm{C} 10^{\mathrm{i}}-\mathrm{N} 2-\mathrm{C} 7-\mathrm{C} 8$

$\mathrm{Ag} 1-\mathrm{N} 2-\mathrm{C} 7-\mathrm{C} 8$

$\mathrm{N} 2-\mathrm{C} 7-\mathrm{C} 10-\mathrm{N} 2^{\mathrm{i}}$

$\mathrm{C} 8-\mathrm{C} 7-\mathrm{C} 10-\mathrm{N} 2^{\mathrm{i}}$

$\mathrm{N} 2-\mathrm{C} 7-\mathrm{C} 10-\mathrm{C} 11$

$\mathrm{C} 8-\mathrm{C} 7-\mathrm{C} 10-\mathrm{C} 11$

$\mathrm{N} 2-\mathrm{C} 10-\mathrm{C} 11-\mathrm{S} 4$

$\mathrm{C} 7-\mathrm{C} 10-\mathrm{C} 11-\mathrm{S} 4$

$\mathrm{C} 12-\mathrm{S} 4-\mathrm{C} 11-\mathrm{C} 10$

$\mathrm{Ag} 1-\mathrm{S} 4-\mathrm{C} 11-\mathrm{C} 10$

$\mathrm{N} 2-\mathrm{C} 7-\mathrm{C} 8-\mathrm{S} 3$

$\mathrm{C} 10-\mathrm{C} 7-\mathrm{C} 8-\mathrm{S} 3$

$\mathrm{C} 9-\mathrm{S} 3-\mathrm{C} 8-\mathrm{C} 7$
109.5

109.5

109.5

109.5

109.5

109.5

109.5

109.5

109.5

109.5

109.5

109.5

$130.3(10)$

134.4 (9)

121.9 (11)

106.9 (8)

$108.2(8)$

$116.2(10)$

$178.5(5)$

$7.0(6)$

$1.3(8)$

$-178.4(5)$

$177.5(5)$

$-2.3(8)$

-41.6 (6)

142.1 (4)

-60.4 (5)

44.3 (4)

107.7 (5)

-72.5 (6)

$-63.6(5)$

Symmetry codes: (i) $-x,-y+1,-z+1$; (ii) $-x+1,-y+1,-z+2$.

Hydrogen-bond geometry $\left(\AA,{ }^{\circ}\right)$

\begin{tabular}{lllll}
\hline$D-\mathrm{H} \cdots A$ & $D-\mathrm{H}$ & $\mathrm{H} \cdots A$ & $D \cdots A$ & $D-\mathrm{H} \cdots A$ \\
\hline $\mathrm{C} 9-\mathrm{H} 9 C \cdots \mathrm{S} 2$ & 0.96 & 2.86 & $3.650(8)$ & 141 \\
$\mathrm{C} 11-\mathrm{H} 11 A \cdots \mathrm{S} 3$ & 0.97 & 2.74 & $3.502(6)$ & 136 \\
$\mathrm{C} 11-\mathrm{H} 11 B \cdots \mathrm{O} 13 A$ & 0.97 & 2.52 & $3.438(17)$ & 157 \\
$\mathrm{C} 2-\mathrm{H} 2 A \cdots \mathrm{O} 11^{\text {iii }}$ & 0.97 & 2.55 & $3.460(9)$ & 156 \\
$\mathrm{C} 2-\mathrm{H} 2 B \cdots \mathrm{O} 12 A^{\text {iv }}$ & 0.97 & 2.53 & $3.431(15)$ & 154 \\
$\mathrm{C} 3-\mathrm{H} 3 C \cdots \mathrm{O} 12 A^{\text {iv }}$ & 0.96 & 2.37 & $3.171(17)$ & 141 \\
$\mathrm{C} 3-\mathrm{H} 3 C \cdots \mathrm{O} 12 B^{\text {iv }}$ & 0.96 & 2.57 & $3.364(16)$ & 140
\end{tabular}




$\begin{array}{llllr}\mathrm{C} 6-\mathrm{H} 6 A \cdots \mathrm{O} 13 A^{\mathrm{i}} & 0.96 & 2.52 & 3.375(19) & 149 \\ \mathrm{C} 9-\mathrm{H} 9 A \cdots \mathrm{O} 11^{\mathrm{v}} & 0.96 & 2.58 & 3.503(10) & 162\end{array}$

Symmetry codes: (i) $-x,-y+1,-z+1$; (iii) $x+1 / 2,-y+1 / 2, z+1 / 2$; (iv) $-x+1,-y+1,-z+1$; (v) $x-1 / 2,-y+1 / 2, z+1 / 2$.

(II) Poly[di- $\mu$-nitrato-bis $\{\mu$-2,3,5,6-tetrakis[(phenylsulfanyl)methyl]pyrazine $\}$ disilver]

\section{Crystal data}

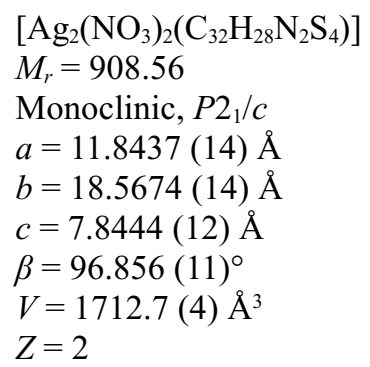

\section{Data collection}

Stoe AED2 4-circle diffractometer

Radiation source: fine-focus sealed tube Graphite monochromator $\omega / 2 \theta$ scans

3178 measured reflections

3178 independent reflections

2606 reflections with $I>2 \sigma(I)$

\section{Refinement}

Refinement on $F^{2}$

Least-squares matrix: full

$R\left[F^{2}>2 \sigma\left(F^{2}\right)\right]=0.045$

$w R\left(F^{2}\right)=0.100$

$S=1.16$

3178 reflections

218 parameters

0 restraints

Primary atom site location: structure-invariant direct methods

Secondary atom site location: difference Fourier map

\section{Special details}

Geometry. All esds (except the esd in the dihedral angle between two 1.s. planes) are estimated using the full covariance matrix. The cell esds are taken into account individually in the estimation of esds in distances, angles and torsion angles; correlations between esds in cell parameters are only used when they are defined by crystal symmetry. An approximate (isotropic) treatment of cell esds is used for estimating esds involving l.s. planes.

Fractional atomic coordinates and isotropic or equivalent isotropic displacement parameters $\left(\AA^{2}\right)$

\begin{tabular}{lllll}
\hline & $x$ & $y$ & $z$ & $U_{\text {iso }} * U_{\text {eq }}$ \\
\hline Ag1 & $1.03726(4)$ & $0.81386(2)$ & $0.31389(6)$ & $0.05335(17)$ \\
S1 & $0.82101(11)$ & $0.85133(7)$ & $0.23374(16)$ & $0.0473(3)$ \\
S2 & $0.75690(11)$ & $1.14870(6)$ & $0.53250(17)$ & $0.0458(3)$
\end{tabular}

$F(000)=908$

$D_{\mathrm{x}}=1.762 \mathrm{Mg} \mathrm{m}^{-3}$

$\theta=11.2-17.7^{\circ}$

$\mu=1.44 \mathrm{~mm}^{-1}$

$T=293 \mathrm{~K}$

$0.46 \times 0.46 \times 0.38 \mathrm{~mm}$
Mo $K \alpha$ radiation, $\lambda=0.71073 \AA$

Block, pale yellow
Hydrogen site location: inferred from neighbouring sites

$\mathrm{H}$-atom parameters constrained

$w=1 /\left[\sigma^{2}\left(F_{\mathrm{o}}^{2}\right)+(0.0308 P)^{2}+3.2342 P\right]$

where $P=\left(F_{\mathrm{o}}{ }^{2}+2 F_{\mathrm{c}}{ }^{2}\right) / 3$

$(\Delta / \sigma)_{\max }=0.001$

$\Delta \rho_{\max }=0.62 \mathrm{e} \AA^{-3}$

$\Delta \rho_{\min }=-0.61$ e $\AA^{-3}$

Extinction correction: SHELXL, $\mathrm{Fc}^{*}=\mathrm{kFc}\left[1+0.001 \mathrm{xFc}^{2} \lambda^{3} / \sin (2 \theta)\right]^{-1 / 4}$

Extinction coefficient: 0.0018 (4)
162 


$\begin{array}{lllll}\text { O1 } & 1.0997(4) & 0.6845(2) & 0.2690(5) & 0.0699(12) \\ \text { O2 } & 1.0189(4) & 0.7981(2) & -0.0056(5) & 0.0671(11) \\ \text { O3 } & 1.0734(4) & 0.5953(2) & 0.4353(5) & 0.0786(13) \\ \text { N1 } & 1.0335(3) & 0.94469(19) & 0.4019(4) & 0.0368(8) \\ \text { N10 } & 1.0647(4) & 0.6592(2) & 0.4005(5) & 0.0464(10) \\ \text { C1 } & 0.9323(4) & 0.9750(2) & 0.3633(5) & 0.0354(10) \\ \text { C2 } & 0.8967(4) & 1.0317(2) & 0.4638(5) & 0.0365(10) \\ \text { C3 } & 0.8593(4) & 0.9458(2) & 0.2087(6) & 0.0435(11) \\ \text { H3A } & 0.8998 & 0.9505 & 0.1089 & 0.052^{*} \\ \text { H3B } & 0.7904 & 0.9743 & 0.1886 & 0.052^{*} \\ \text { C4 } & 0.7519(4) & 0.8528(3) & 0.4246(6) & 0.0446(11) \\ \text { C5 } & 0.8133(5) & 0.8402(3) & 0.5828(7) & 0.0529(13) \\ \text { H5 } & 0.8919 & 0.8349 & 0.5915 & 0.063^{*} \\ \text { C6 } & 0.7584(6) & 0.8354(3) & 0.7273(7) & 0.0625(15) \\ \text { H6 } & 0.8002 & 0.8266 & 0.8332 & 0.075^{*} \\ \text { C7 } & 0.6437(6) & 0.8435(4) & 0.7171(9) & 0.0738(18) \\ \text { H7 } & 0.6072 & 0.8402 & 0.8154 & 0.089^{*} \\ \text { C8 } & 0.5828(6) & 0.8564(5) & 0.5616(10) & 0.091(2) \\ \text { H8 } & 0.5044 & 0.8621 & 0.5545 & 0.110^{*} \\ \text { C9 } & 0.6358(5) & 0.8613(4) & 0.4139(9) & 0.0767(19) \\ \text { H9 } & 0.5933 & 0.8701 & 0.3084 & 0.092^{*} \\ \text { C10 } & 0.7834(4) & 1.0681(2) & 0.4170(6) & 0.0406(10) \\ \text { H10A } & 0.7241 & 1.0337 & 0.4334 & 0.049^{*} \\ \text { H10B } & 0.7763 & 1.0797 & 0.2957 & 0.049^{*} \\ \text { C11 } & 0.7013(4) & 1.1194(3) & 0.7201(6) & 0.0439(11) \\ \text { C12 } & 0.6681(5) & 1.1731(3) & 0.8251(7) & 0.0588(14) \\ \text { H12 } & 0.6784 & 1.2211 & 0.7969 & 0.071^{*} \\ \text { C13 } & 0.6194(5) & 1.1561(4) & 0.9719(8) & 0.0700(17) \\ \text { H13 } & 0.5977 & 1.1926 & 1.0422 & 0.084^{*} \\ \text { C14 } & 0.6031(5) & 1.0855(4) & 1.0141(7) & 0.0710(19) \\ \text { H14 } & 0.5694 & 1.0742 & 1.1119 & 0.085^{*} \\ \text { C15 } & 0.6368(5) & 1.0312(4) & 0.9113(7) & 0.0646(16) \\ \text { H15 } & 0.6262 & 0.9832 & 0.9401 & 0.078^{*} \\ \text { C16 } & 0.6866(5) & 1.0481(3) & 0.7647(7) & 0.0539(13) \\ \text { H16 } & 0.7101 & 1.0115 & 0.6963 & 0.065^{*} \\ & & & & \end{array}$

Atomic displacement parameters $\left(\AA^{2}\right)$

\begin{tabular}{lllllll}
\hline & $U^{11}$ & $U^{22}$ & $U^{33}$ & $U^{12}$ & $U^{13}$ & $U^{23}$ \\
\hline Ag1 & $0.0616(3)$ & $0.0377(2)$ & $0.0624(3)$ & $0.00179(19)$ & $0.01433(19)$ & $-0.00855(18)$ \\
S1 & $0.0601(8)$ & $0.0426(7)$ & $0.0388(6)$ & $-0.0055(6)$ & $0.0047(6)$ & $-0.0088(5)$ \\
S2 & $0.0516(7)$ & $0.0354(6)$ & $0.0513(7)$ & $0.0076(5)$ & $0.0109(6)$ & $0.0029(5)$ \\
O1 & $0.107(3)$ & $0.057(2)$ & $0.052(2)$ & $0.015(2)$ & $0.034(2)$ & $0.0080(19)$ \\
O2 & $0.097(3)$ & $0.057(2)$ & $0.052(2)$ & $-0.019(2)$ & $0.029(2)$ & $-0.0007(18)$ \\
O3 & $0.130(4)$ & $0.040(2)$ & $0.070(3)$ & $0.010(2)$ & $0.024(3)$ & $0.0085(19)$ \\
N1 & $0.047(2)$ & $0.0323(19)$ & $0.0303(19)$ & $0.0022(17)$ & $0.0034(16)$ & $-0.0011(15)$ \\
N10 & $0.058(3)$ & $0.041(2)$ & $0.041(2)$ & $0.003(2)$ & $0.0092(19)$ & $0.0019(19)$ \\
C1 & $0.047(3)$ & $0.028(2)$ & $0.031(2)$ & $-0.0015(19)$ & $0.0042(19)$ & $0.0014(17)$
\end{tabular}




$\begin{array}{lllllll}\text { C2 } & 0.047(3) & 0.031(2) & 0.032(2) & -0.003(2) & 0.006(2) & 0.0035(18) \\ \text { C3 } & 0.059(3) & 0.037(2) & 0.032(2) & 0.003(2) & -0.003(2) & 0.0010(19) \\ \text { C4 } & 0.050(3) & 0.040(3) & 0.044(3) & -0.003(2) & 0.005(2) & -0.002(2) \\ \text { C5 } & 0.046(3) & 0.062(3) & 0.052(3) & 0.009(3) & 0.009(2) & 0.006(3) \\ \text { C6 } & 0.078(4) & 0.064(4) & 0.048(3) & 0.011(3) & 0.015(3) & 0.006(3) \\ \text { C7 } & 0.079(5) & 0.080(4) & 0.069(4) & -0.001(4) & 0.035(4) & -0.002(3) \\ \text { C8 } & 0.049(4) & 0.132(7) & 0.096(6) & -0.002(4) & 0.022(4) & -0.005(5) \\ \text { C9 } & 0.054(4) & 0.109(6) & 0.065(4) & -0.003(4) & -0.005(3) & -0.004(4) \\ \text { C10 } & 0.048(3) & 0.036(2) & 0.037(2) & 0.002(2) & 0.004(2) & 0.000(2) \\ \text { C11 } & 0.040(3) & 0.050(3) & 0.043(3) & -0.001(2) & 0.006(2) & -0.003(2) \\ \text { C12 } & 0.065(4) & 0.056(3) & 0.057(3) & 0.002(3) & 0.013(3) & -0.017(3) \\ \text { C13 } & 0.066(4) & 0.090(5) & 0.055(4) & 0.002(4) & 0.011(3) & -0.019(3) \\ \text { C14 } & 0.052(3) & 0.122(6) & 0.041(3) & -0.012(4) & 0.010(3) & 0.001(3) \\ \text { C15 } & 0.060(4) & 0.079(4) & 0.055(3) & -0.016(3) & 0.009(3) & 0.012(3) \\ \text { C16 } & 0.060(3) & 0.052(3) & 0.051(3) & -0.002(3) & 0.013(3) & -0.001(2)\end{array}$

Geometric parameters $\left(\hat{A},{ }^{o}\right)$

\begin{tabular}{|c|c|c|c|}
\hline $\operatorname{Ag} 1-\mathrm{N} 1$ & $2.527(4)$ & $\mathrm{C} 4-\mathrm{C} 9$ & $1.376(8)$ \\
\hline $\mathrm{Ag} 1-\mathrm{S} 1$ & $2.6560(15)$ & $\mathrm{C} 4-\mathrm{C} 5$ & $1.382(7)$ \\
\hline $\mathrm{Ag} 1-\mathrm{S} 2^{\mathrm{i}}$ & $2.6790(14)$ & $\mathrm{C} 5-\mathrm{C} 6$ & $1.376(7)$ \\
\hline $\mathrm{Ag} 1-\mathrm{O} 1$ & $2.551(4)$ & $\mathrm{C} 5-\mathrm{H} 5$ & 0.9300 \\
\hline $\mathrm{Ag} 1-\mathrm{O} 2$ & $2.507(4)$ & $\mathrm{C} 6-\mathrm{C} 7$ & $1.359(9)$ \\
\hline $\mathrm{Ag} 1-\mathrm{O} 2^{\mathrm{ii}}$ & $2.539(4)$ & C6- $\mathrm{H} 6$ & 0.9300 \\
\hline $\mathrm{S} 1-\mathrm{C} 4$ & $1.790(5)$ & $\mathrm{C} 7-\mathrm{C} 8$ & $1.363(10)$ \\
\hline $\mathrm{S} 1-\mathrm{C} 3$ & $1.828(5)$ & $\mathrm{C} 7-\mathrm{H} 7$ & 0.9300 \\
\hline $\mathrm{S} 2-\mathrm{C} 11$ & $1.768(5)$ & $\mathrm{C} 8-\mathrm{C} 9$ & $1.385(9)$ \\
\hline $\mathrm{S} 2-\mathrm{C} 10$ & $1.796(4)$ & $\mathrm{C} 8-\mathrm{H} 8$ & 0.9300 \\
\hline $\mathrm{S} 2-\mathrm{Ag} 1^{\mathrm{i}}$ & $2.6790(14)$ & $\mathrm{C} 9-\mathrm{H} 9$ & 0.9300 \\
\hline $\mathrm{O} 1-\mathrm{N} 10$ & $1.248(5)$ & $\mathrm{C} 10-\mathrm{H} 10 \mathrm{~A}$ & 0.9700 \\
\hline $\mathrm{O} 2-\mathrm{N} 10^{\mathrm{iii}}$ & $1.250(5)$ & $\mathrm{C} 10-\mathrm{H} 10 \mathrm{~B}$ & 0.9700 \\
\hline $\mathrm{O} 2-\mathrm{Ag} 1^{\mathrm{iii}}$ & $2.539(4)$ & $\mathrm{C} 11-\mathrm{C} 12$ & $1.381(7)$ \\
\hline $\mathrm{O} 3-\mathrm{N} 10$ & $1.219(5)$ & $\mathrm{C} 11-\mathrm{C} 16$ & $1.385(7)$ \\
\hline $\mathrm{N} 1-\mathrm{C} 1$ & $1.326(6)$ & $\mathrm{C} 12-\mathrm{C} 13$ & $1.385(8)$ \\
\hline $\mathrm{N} 1-\mathrm{C} 2^{\mathrm{i}}$ & $1.334(5)$ & $\mathrm{C} 12-\mathrm{H} 12$ & 0.9300 \\
\hline $\mathrm{N} 10-\mathrm{O} 2^{\mathrm{ii}}$ & $1.250(5)$ & $\mathrm{C} 13-\mathrm{C} 14$ & $1.372(9)$ \\
\hline $\mathrm{C} 1-\mathrm{C} 2$ & $1.409(6)$ & $\mathrm{C} 13-\mathrm{H} 13$ & 0.9300 \\
\hline $\mathrm{C} 1-\mathrm{C} 3$ & $1.504(6)$ & $\mathrm{C} 14-\mathrm{C} 15$ & $1.380(9)$ \\
\hline $\mathrm{C} 2-\mathrm{N} 1^{\mathrm{i}}$ & $1.334(5)$ & $\mathrm{C} 14-\mathrm{H} 14$ & 0.9300 \\
\hline $\mathrm{C} 2-\mathrm{C} 10$ & $1.508(6)$ & $\mathrm{C} 15-\mathrm{C} 16$ & $1.390(7)$ \\
\hline $\mathrm{C} 3-\mathrm{H} 3 \mathrm{~A}$ & 0.9700 & $\mathrm{C} 15-\mathrm{H} 15$ & 0.9300 \\
\hline $\mathrm{C} 3-\mathrm{H} 3 \mathrm{~B}$ & 0.9700 & $\mathrm{C} 16-\mathrm{H} 16$ & 0.9300 \\
\hline $\mathrm{N} 1-\mathrm{Ag} 1-\mathrm{S} 1$ & $76.40(9)$ & $\mathrm{C} 9-\mathrm{C} 4-\mathrm{C} 5$ & $119.3(5)$ \\
\hline $\mathrm{N} 1-\mathrm{Ag} 1-\mathrm{S} 2^{\mathrm{i}}$ & $70.89(9)$ & $\mathrm{C} 9-\mathrm{C} 4-\mathrm{S} 1$ & $120.3(4)$ \\
\hline $\mathrm{S} 1-\mathrm{Ag} 1-\mathrm{S} 2^{\mathrm{i}}$ & $146.98(4)$ & $\mathrm{C} 5-\mathrm{C} 4-\mathrm{S} 1$ & $120.2(4)$ \\
\hline $\mathrm{O} 2-\mathrm{Ag} 1-\mathrm{N} 1$ & $112.54(12)$ & $\mathrm{C} 6-\mathrm{C} 5-\mathrm{C} 4$ & $120.1(5)$ \\
\hline $\mathrm{O} 2-\mathrm{Ag} 1-\mathrm{O} 2^{\mathrm{ii}}$ & $117.32(8)$ & $\mathrm{C} 6-\mathrm{C} 5-\mathrm{H} 5$ & 120.0 \\
\hline
\end{tabular}




\begin{tabular}{|c|c|c|c|}
\hline $\mathrm{N} 1-\mathrm{Ag} 1-\mathrm{O} 2^{\mathrm{ii}}$ & $128.98(12)$ & $\mathrm{C} 4-\mathrm{C} 5-\mathrm{H} 5$ & 120.0 \\
\hline $\mathrm{O} 2-\mathrm{Ag} 1-\mathrm{O} 1$ & $75.15(13)$ & $\mathrm{C} 7-\mathrm{C} 6-\mathrm{C} 5$ & $120.8(6)$ \\
\hline $\mathrm{N} 1-\mathrm{Ag} 1-\mathrm{O} 1$ & $163.34(14)$ & $\mathrm{C} 7-\mathrm{C} 6-\mathrm{H} 6$ & 119.6 \\
\hline $\mathrm{O} 2^{\mathrm{ii}}-\mathrm{Ag} 1-\mathrm{O} 1$ & $49.56(12)$ & $\mathrm{C} 5-\mathrm{C} 6-\mathrm{H} 6$ & 119.6 \\
\hline $\mathrm{O} 2-\mathrm{Ag} 1-\mathrm{S} 1$ & $80.10(11)$ & $\mathrm{C} 6-\mathrm{C} 7-\mathrm{C} 8$ & $119.4(6)$ \\
\hline $\mathrm{O} 2^{\mathrm{ii}}-\mathrm{Ag} 1-\mathrm{S} 1$ & $101.67(11)$ & $\mathrm{C} 6-\mathrm{C} 7-\mathrm{H} 7$ & 120.3 \\
\hline $\mathrm{O} 1-\mathrm{Ag} 1-\mathrm{S} 1$ & $120.09(11)$ & $\mathrm{C} 8-\mathrm{C} 7-\mathrm{H} 7$ & 120.3 \\
\hline $\mathrm{O} 2-\mathrm{Ag} 1-\mathrm{S} 2^{\mathrm{i}}$ & $116.46(10)$ & $\mathrm{C} 7-\mathrm{C} 8-\mathrm{C} 9$ & $121.1(6)$ \\
\hline $\mathrm{O} 2^{\mathrm{ii}}-\mathrm{Ag} 1-\mathrm{S} 2^{\mathrm{i}}$ & $95.47(11)$ & $\mathrm{C} 7-\mathrm{C} 8-\mathrm{H} 8$ & 119.5 \\
\hline $\mathrm{O} 1-\mathrm{Ag} 1-\mathrm{S} 2^{\mathrm{i}}$ & $92.47(11)$ & $\mathrm{C} 9-\mathrm{C} 8-\mathrm{H} 8$ & 119.5 \\
\hline $\mathrm{C} 4-\mathrm{S} 1-\mathrm{C} 3$ & $102.6(2)$ & $\mathrm{C} 4-\mathrm{C} 9-\mathrm{C} 8$ & $119.4(6)$ \\
\hline $\mathrm{C} 4-\mathrm{S} 1-\mathrm{Ag} 1$ & $109.31(17)$ & $\mathrm{C} 4-\mathrm{C} 9-\mathrm{H} 9$ & 120.3 \\
\hline $\mathrm{C} 3-\mathrm{S} 1-\mathrm{Ag} 1$ & $91.85(17)$ & $\mathrm{C} 8-\mathrm{C} 9-\mathrm{H} 9$ & 120.3 \\
\hline $\mathrm{C} 11-\mathrm{S} 2-\mathrm{C} 10$ & $105.6(2)$ & $\mathrm{C} 2-\mathrm{C} 10-\mathrm{S} 2$ & $117.1(3)$ \\
\hline $\mathrm{C} 11-\mathrm{S} 2-\mathrm{Ag} 1^{\mathrm{i}}$ & $96.58(16)$ & $\mathrm{C} 2-\mathrm{C} 10-\mathrm{H} 10 \mathrm{~A}$ & 108.0 \\
\hline $\mathrm{C} 10-\mathrm{S} 2-\mathrm{Ag} 1^{\mathrm{i}}$ & $103.79(16)$ & $\mathrm{S} 2-\mathrm{C} 10-\mathrm{H} 10 \mathrm{~A}$ & 108.0 \\
\hline $\mathrm{N} 10-\mathrm{O} 1-\mathrm{Ag} 1$ & $96.3(3)$ & $\mathrm{C} 2-\mathrm{C} 10-\mathrm{H} 10 \mathrm{~B}$ & 108.0 \\
\hline $\mathrm{N} 10^{\mathrm{iii}}-\mathrm{O} 2-\mathrm{Ag} 1$ & $121.5(3)$ & $\mathrm{S} 2-\mathrm{C} 10-\mathrm{H} 10 \mathrm{~B}$ & 108.0 \\
\hline $\mathrm{N} 10^{\mathrm{iii}}-\mathrm{O} 2-\mathrm{Ag} 1^{\mathrm{iii}}$ & $96.8(3)$ & $\mathrm{H} 10 \mathrm{~A}-\mathrm{C} 10-\mathrm{H} 10 \mathrm{~B}$ & 107.3 \\
\hline $\mathrm{Ag} 1-\mathrm{O} 2-\mathrm{Ag} 1^{\mathrm{iii}}$ & $130.58(17)$ & $\mathrm{C} 12-\mathrm{C} 11-\mathrm{C} 16$ & $119.1(5)$ \\
\hline $\mathrm{C} 1-\mathrm{N} 1-\mathrm{C} 2^{\mathrm{i}}$ & $119.9(4)$ & $\mathrm{C} 12-\mathrm{C} 11-\mathrm{S} 2$ & $115.8(4)$ \\
\hline $\mathrm{C} 1-\mathrm{N} 1-\mathrm{Ag} 1$ & $113.0(3)$ & $\mathrm{C} 16-\mathrm{C} 11-\mathrm{S} 2$ & $125.1(4)$ \\
\hline $\mathrm{C} 2{ }^{\mathrm{i}}-\mathrm{N} 1-\mathrm{Ag} 1$ & $120.2(3)$ & $\mathrm{C} 11-\mathrm{C} 12-\mathrm{C} 13$ & $120.5(6)$ \\
\hline $\mathrm{O} 3-\mathrm{N} 10-\mathrm{O} 1$ & $121.6(4)$ & $\mathrm{C} 11-\mathrm{C} 12-\mathrm{H} 12$ & 119.8 \\
\hline $\mathrm{O} 3-\mathrm{N} 10-\mathrm{O} 2^{\mathrm{ii}}$ & $121.1(4)$ & $\mathrm{C} 13-\mathrm{C} 12-\mathrm{H} 12$ & 119.8 \\
\hline $\mathrm{O} 1-\mathrm{N} 10-\mathrm{O} 2^{\mathrm{ii}}$ & $117.3(4)$ & $\mathrm{C} 14-\mathrm{C} 13-\mathrm{C} 12$ & $120.3(6)$ \\
\hline $\mathrm{N} 1-\mathrm{C} 1-\mathrm{C} 2$ & $120.8(4)$ & $\mathrm{C} 14-\mathrm{C} 13-\mathrm{H} 13$ & 119.9 \\
\hline $\mathrm{N} 1-\mathrm{C} 1-\mathrm{C} 3$ & $116.6(4)$ & $\mathrm{C} 12-\mathrm{C} 13-\mathrm{H} 13$ & 119.9 \\
\hline $\mathrm{C} 2-\mathrm{C} 1-\mathrm{C} 3$ & $122.6(4)$ & $\mathrm{C} 13-\mathrm{C} 14-\mathrm{C} 15$ & $119.9(5)$ \\
\hline $\mathrm{N} 1-\mathrm{C} 2-\mathrm{C} 1$ & $119.2(4)$ & $\mathrm{C} 13-\mathrm{C} 14-\mathrm{H} 14$ & 120.0 \\
\hline $\mathrm{N} 1-\mathrm{C} 2-\mathrm{C} 10$ & $119.6(4)$ & $\mathrm{C} 15-\mathrm{C} 14-\mathrm{H} 14$ & 120.0 \\
\hline $\mathrm{C} 1-\mathrm{C} 2-\mathrm{C} 10$ & $121.1(4)$ & $\mathrm{C} 14-\mathrm{C} 15-\mathrm{C} 16$ & $119.9(6)$ \\
\hline $\mathrm{C} 1-\mathrm{C} 3-\mathrm{S} 1$ & $112.7(3)$ & $\mathrm{C} 14-\mathrm{C} 15-\mathrm{H} 15$ & 120.0 \\
\hline $\mathrm{C} 1-\mathrm{C} 3-\mathrm{H} 3 \mathrm{~A}$ & 109.1 & $\mathrm{C} 16-\mathrm{C} 15-\mathrm{H} 15$ & 120.0 \\
\hline $\mathrm{S} 1-\mathrm{C} 3-\mathrm{H} 3 \mathrm{~A}$ & 109.1 & $\mathrm{C} 11-\mathrm{C} 16-\mathrm{C} 15$ & $120.3(5)$ \\
\hline $\mathrm{C} 1-\mathrm{C} 3-\mathrm{H} 3 \mathrm{~B}$ & 109.1 & $\mathrm{C} 11-\mathrm{C} 16-\mathrm{H} 16$ & 119.8 \\
\hline $\mathrm{S} 1-\mathrm{C} 3-\mathrm{H} 3 \mathrm{~B}$ & 109.1 & $\mathrm{C} 15-\mathrm{C} 16-\mathrm{H} 16$ & 119.8 \\
\hline $\mathrm{H} 3 \mathrm{~A}-\mathrm{C} 3-\mathrm{H} 3 \mathrm{~B}$ & 107.8 & & \\
\hline $\mathrm{Ag} 1-\mathrm{O} 1-\mathrm{N} 10-\mathrm{O} 3$ & $178.4(5)$ & $\mathrm{C} 5-\mathrm{C} 6-\mathrm{C} 7-\mathrm{C} 8$ & $0.0(10)$ \\
\hline $\mathrm{Ag} 1-\mathrm{O} 1-\mathrm{N} 10-\mathrm{O} 2^{\mathrm{ii}}$ & $-0.7(5)$ & $\mathrm{C} 6-\mathrm{C} 7-\mathrm{C} 8-\mathrm{C} 9$ & $0.2(12)$ \\
\hline $\mathrm{C} 22^{\mathrm{i}-\mathrm{N} 1-\mathrm{C} 1-\mathrm{C} 2}$ & $-0.7(7)$ & $\mathrm{C} 5-\mathrm{C} 4-\mathrm{C} 9-\mathrm{C} 8$ & $-0.4(10)$ \\
\hline $\mathrm{Ag} 1-\mathrm{N} 1-\mathrm{C} 1-\mathrm{C} 2$ & $150.3(3)$ & $\mathrm{S} 1-\mathrm{C} 4-\mathrm{C} 9-\mathrm{C} 8$ & $174.8(6)$ \\
\hline $\mathrm{C} 2-\mathrm{i} 1-\mathrm{C} 1-\mathrm{C} 3$ & $179.3(4)$ & $\mathrm{C} 7-\mathrm{C} 8-\mathrm{C} 9-\mathrm{C} 4$ & $0.0(12)$ \\
\hline $\mathrm{Ag} 1-\mathrm{N} 1-\mathrm{C} 1-\mathrm{C} 3$ & $-29.7(5)$ & $\mathrm{N} 1{ }^{\mathrm{i}}-\mathrm{C} 2-\mathrm{C} 10-\mathrm{S} 2$ & $7.1(6)$ \\
\hline $\mathrm{N} 1-\mathrm{C} 1-\mathrm{C} 2-\mathrm{N} 1^{\mathrm{i}}$ & $0.7(7)$ & $\mathrm{C} 1-\mathrm{C} 2-\mathrm{C} 10-\mathrm{S} 2$ & $-170.1(3)$ \\
\hline $\mathrm{C} 3-\mathrm{C} 1-\mathrm{C} 2-\mathrm{N} 1^{\mathrm{i}}$ & $-179.3(4)$ & $\mathrm{C} 11-\mathrm{S} 2-\mathrm{C} 10-\mathrm{C} 2$ & $-86.4(4)$ \\
\hline $\mathrm{N} 1-\mathrm{C} 1-\mathrm{C} 2-\mathrm{C} 10$ & $177.9(4)$ & $\mathrm{Ag} 11^{\mathrm{i}}-\mathrm{S} 2-\mathrm{C} 10-\mathrm{C} 2$ & $14.6(4)$ \\
\hline
\end{tabular}




$\begin{array}{llll}\mathrm{C} 3-\mathrm{C} 1-\mathrm{C} 2-\mathrm{C} 10 & -2.2(6) & \mathrm{C} 10-\mathrm{S} 2-\mathrm{C} 11-\mathrm{C} 12 & -176.3(4) \\ \mathrm{N} 1-\mathrm{C} 1-\mathrm{C} 3-\mathrm{S} 1 & 62.9(5) & \mathrm{Ag} 1-\mathrm{S} 2-\mathrm{C} 11-\mathrm{C} 12 & 77.3(4) \\ \mathrm{C} 2-\mathrm{C} 1-\mathrm{C} 3-\mathrm{S} 1 & -117.1(4) & \mathrm{C} 10-\mathrm{S} 2-\mathrm{C} 11-\mathrm{C} 16 & 1.8(5) \\ \mathrm{C} 4-\mathrm{S} 1-\mathrm{C} 3-\mathrm{C} 1 & 57.2(4) & \mathrm{Ag} 1-\mathrm{S} 2-\mathrm{C} 11-\mathrm{C} 16 & -104.5(5) \\ \mathrm{Ag} 1-\mathrm{S} 1-\mathrm{C} 3-\mathrm{C} 1 & -53.1(3) & \mathrm{C} 16-\mathrm{C} 11-\mathrm{C} 12-\mathrm{C} 13 & -0.7(8) \\ \mathrm{C} 3-\mathrm{S} 1-\mathrm{C} 4-\mathrm{C} 9 & 92.2(5) & \mathrm{S} 2-\mathrm{C} 11-\mathrm{C} 12-\mathrm{C} 13 & 177.6(5) \\ \mathrm{Ag} 1-\mathrm{S} 1-\mathrm{C} 4-\mathrm{C} 9 & -171.3(5) & \mathrm{C} 11-\mathrm{C} 12-\mathrm{C} 13-\mathrm{C} 14 & -0.4(9) \\ \mathrm{C} 3-\mathrm{S} 1-\mathrm{C} 4-\mathrm{C} 5 & -92.6(5) & \mathrm{C} 12-\mathrm{C} 13-\mathrm{C} 14-\mathrm{C} 15 & 1.0(10) \\ \mathrm{Ag} 1-\mathrm{S} 1-\mathrm{C} 4-\mathrm{C} 5 & 3.9(5) & \mathrm{C} 13-\mathrm{C} 14-\mathrm{C} 15-\mathrm{C} 16 & -0.4(9) \\ \mathrm{C} 9-\mathrm{C} 4-\mathrm{C} 5-\mathrm{C} 6 & 0.6(9) & \mathrm{C} 12-\mathrm{C} 11-\mathrm{C} 16-\mathrm{C} 15 & 1.3(8) \\ \mathrm{S} 1-\mathrm{C} 4-\mathrm{C} 5-\mathrm{C} 6 & -174.6(4) & \mathrm{S} 2-\mathrm{C} 11-\mathrm{C} 16-\mathrm{C} 15 & -176.8(4) \\ \mathrm{C} 4-\mathrm{C} 5-\mathrm{C} 6-\mathrm{C} 7 & -0.4(9) & \mathrm{C} 14-\mathrm{C} 15-\mathrm{C} 16-\mathrm{C} 11 & -0.8(9)\end{array}$

Symmetry codes: (i) $-x+2,-y+2,-z+1$; (ii) $x,-y+3 / 2, z+1 / 2$; (iii) $x,-y+3 / 2, z-1 / 2$.

(III) Poly[[trinitrato $\left\{\mu_{6}-2,3,5,6\right.$-tetrakis[(pyridin-2-ylsulfanyl)methyl]pyrazine\}trisilver(I)]

Crystal data

$\left[\mathrm{Ag}_{3}\left(\mathrm{NO}_{3}\right)_{3}\left(\mathrm{C}_{28} \mathrm{H}_{24} \mathrm{~N}_{6} \mathrm{~S}_{4}\right)\right]$

$M_{r}=1082.41$

Monoclinic, $C 2 / c$

$a=13.6319(9) \AA$

$b=16.2211(10) \AA$

$c=15.7201(11) \AA$

$\beta=96.607(8)^{\circ}$

$V=3453.0(4) \AA^{3}$

$Z=4$

\section{Data collection}

STOE IPDS 1

diffractometer

Radiation source: fine-focus sealed tube

Plane graphite monochromator

$\varphi$ rotation scans

Absorption correction: multi-scan

(MULABS; Spek, 2009)

$T_{\text {min }}=0.949, T_{\text {max }}=1.000$

\section{Refinement}

Refinement on $F^{2}$

Least-squares matrix: full

$R\left[F^{2}>2 \sigma\left(F^{2}\right)\right]=0.030$

$w R\left(F^{2}\right)=0.052$

$S=0.76$

3311 reflections

242 parameters

0 restraints

Primary atom site location: structure-invariant direct methods

Secondary atom site location: difference Fourier map
$F(000)=2128$

$D_{\mathrm{x}}=2.082 \mathrm{Mg} \mathrm{m}^{-3}$

Mo $K \alpha$ radiation, $\lambda=0.71073 \AA$

Cell parameters from 5000 reflections

$\theta=2.0-25.9^{\circ}$

$\mu=1.99 \mathrm{~mm}^{-1}$

$T=223 \mathrm{~K}$

Needle, pale yellow

$0.45 \times 0.08 \times 0.08 \mathrm{~mm}$

13264 measured reflections

3311 independent reflections

1936 reflections with $I>2 \sigma(I)$

$R_{\text {int }}=0.072$

$\theta_{\text {max }}=25.9^{\circ}, \theta_{\min }=2.0^{\circ}$

$h=-16 \rightarrow 16$

$k=-19 \rightarrow 19$

$l=-19 \rightarrow 18$

Hydrogen site location: inferred from neighbouring sites

$\mathrm{H}$-atom parameters constrained

$w=1 /\left[\sigma^{2}\left(F_{\mathrm{o}}{ }^{2}\right)+(0.0179 P)^{2}\right]$

where $P=\left(F_{\mathrm{o}}{ }^{2}+2 F_{\mathrm{c}}{ }^{2}\right) / 3$

$(\Delta / \sigma)_{\max }<0.001$

$\Delta \rho_{\max }=0.43 \mathrm{e} \AA^{-3}$

$\Delta \rho_{\min }=-0.44$ e $\AA^{-3}$

Extinction correction: SHELXL,

$\mathrm{Fc}^{*}=\mathrm{kFc}\left[1+0.001 \mathrm{xFc}^{2} \lambda^{3} / \sin (2 \theta)\right]^{-1 / 4}$

Extinction coefficient: 0.00014 (3) 


\section{Special details}

Geometry. All esds (except the esd in the dihedral angle between two 1.s. planes) are estimated using the full covariance matrix. The cell esds are taken into account individually in the estimation of esds in distances, angles and torsion angles; correlations between esds in cell parameters are only used when they are defined by crystal symmetry. An approximate (isotropic) treatment of cell esds is used for estimating esds involving l.s. planes.

Fractional atomic coordinates and isotropic or equivalent isotropic displacement parameters $\left(\AA^{2}\right)$

\begin{tabular}{|c|c|c|c|c|}
\hline & $x$ & $y$ & $z$ & $U_{\text {iso }} * / U_{\text {eq }}$ \\
\hline $\operatorname{Ag} 1$ & $0.01942(2)$ & $0.37808(2)$ & -0.08024 & $0.04096(12)$ \\
\hline $\mathrm{Ag} 2$ & 0.5000 & -0.03325 & 0.2500 & $0.05015(18)$ \\
\hline S1 & $0.09150(7)$ & $0.43818(6)$ & $0.08153(8)$ & $0.0309(3)$ \\
\hline S2 & $0.37324(7)$ & $0.12904(6)$ & $0.20654(7)$ & $0.0293(3)$ \\
\hline O11 & $0.0033(3)$ & $0.2151(3)$ & $-0.1140(3)$ & $0.0993(17)$ \\
\hline $\mathrm{O} 12$ & $0.0505(3)$ & $0.1241(2)$ & $-0.0186(3)$ & $0.0881(13)$ \\
\hline $\mathrm{O} 13$ & $-0.0038(3)$ & $0.2406(3)$ & $0.0181(3)$ & $0.0871(14)$ \\
\hline $\mathrm{O} 21$ & 0.5000 & $-0.1915(3)$ & 0.2500 & $0.082(2)$ \\
\hline $\mathrm{O} 22$ & $0.5755(3)$ & -0.3025 & $0.2419(3)$ & $0.0890(15)$ \\
\hline N1 & $0.1890(2)$ & $0.31443(19)$ & $-0.0280(2)$ & $0.0228(8)$ \\
\hline $\mathrm{N} 2$ & $0.1418(2)$ & $0.59202(19)$ & $0.0729(2)$ & $0.0278(8)$ \\
\hline N3 & $0.3466(2)$ & $-0.0278(2)$ & $0.1875(2)$ & $0.0277(8)$ \\
\hline N11 & $0.0173(3)$ & $0.1915(3)$ & $-0.0375(4)$ & $0.0643(14)$ \\
\hline $\mathrm{N} 21$ & 0.5000 & $-0.2669(3)$ & 0.2500 & $0.0365(13)$ \\
\hline $\mathrm{C} 1$ & $0.2093(3)$ & $0.2984(2)$ & $0.0553(3)$ & $0.0232(9)$ \\
\hline $\mathrm{C} 2$ & $0.2715(3)$ & $0.2341(2)$ & $0.0833(3)$ & $0.0217(9)$ \\
\hline $\mathrm{C} 3$ & $0.1631(3)$ & 0.3497 (2) & $0.1204(3)$ & $0.0306(11)$ \\
\hline $\mathrm{H} 3 \mathrm{~A}$ & 0.2161 & 0.3689 & 0.1632 & $0.037^{*}$ \\
\hline $\mathrm{H} 3 \mathrm{~B}$ & 0.1204 & 0.3136 & 0.1499 & $0.037^{*}$ \\
\hline $\mathrm{C} 4$ & $0.1816(3)$ & $0.5165(2)$ & $0.0793(3)$ & $0.0295(10)$ \\
\hline $\mathrm{C} 5$ & $0.2821(3)$ & $0.5034(3)$ & $0.0844(3)$ & $0.0517(14)$ \\
\hline H5 & 0.3082 & 0.4497 & 0.0867 & $0.062 *$ \\
\hline C6 & $0.3433(3)$ & $0.5714(3)$ & $0.0861(4)$ & $0.0617(16)$ \\
\hline H6 & 0.4122 & 0.5645 & 0.0912 & $0.074 *$ \\
\hline $\mathrm{C} 7$ & $0.3033(3)$ & $0.6488(3)$ & $0.0803(3)$ & $0.0518(14)$ \\
\hline $\mathrm{H} 7$ & 0.3442 & 0.6956 & 0.0814 & $0.062^{*}$ \\
\hline $\mathrm{C} 8$ & $0.2039(3)$ & $0.6569(3)$ & 0.0731 & $0.0407(12)$ \\
\hline $\mathrm{H} 8$ & 0.1768 & 0.7102 & 0.0679 & $0.049^{*}$ \\
\hline C9 & $0.2962(3)$ & $0.2171(2)$ & $0.1786(3)$ & $0.0279(10)$ \\
\hline H9A & 0.2341 & 0.2099 & 0.2036 & $0.034 *$ \\
\hline H9B & 0.3290 & 0.2660 & 0.2053 & $0.034^{*}$ \\
\hline $\mathrm{C} 11$ & $0.2968(3)$ & $-0.0982(3)$ & 0.1679 (3) & $0.0406(12)$ \\
\hline H11 & 0.3317 & -0.1483 & 0.1722 & $0.049^{*}$ \\
\hline $\mathrm{C} 12$ & $0.1982(4)$ & $-0.0998(3)$ & $0.1421(3)$ & $0.0568(15)$ \\
\hline H12 & 0.1653 & -0.1501 & 0.1296 & $0.068^{*}$ \\
\hline $\mathrm{C} 13$ & $0.1476(4)$ & $-0.0263(3)$ & 0.1347 (4) & $0.0654(17)$ \\
\hline H13 & 0.0792 & -0.0260 & 0.1174 & $0.079^{*}$ \\
\hline $\mathrm{C} 14$ & $0.1966(3)$ & 0.0468 & $0.1524(3)$ & $0.0492(13)$ \\
\hline H14 & 0.1631 & 0.0976 & 0.1466 & $0.059 *$ \\
\hline
\end{tabular}


Atomic displacement parameters $\left(\AA^{2}\right)$

\begin{tabular}{lllllll}
\hline & $U^{11}$ & $U^{22}$ & $U^{33}$ & $U^{12}$ & $U^{13}$ & $U^{23}$ \\
\hline $\mathrm{Ag} 1$ & $0.02369(17)$ & $0.0330(2)$ & $0.0673(3)$ & $0.00667(16)$ & $0.01013(16)$ & $0.0075(2)$ \\
$\mathrm{Ag} 2$ & $0.0223(3)$ & $0.0276(3)$ & $0.0975(5)$ & 0.000 & $-0.0062(3)$ & 0.000 \\
$\mathrm{~S} 1$ & $0.0246(5)$ & $0.0246(6)$ & $0.0447(7)$ & $0.0066(5)$ & $0.0092(5)$ & $0.0023(5)$ \\
$\mathrm{S} 2$ & $0.0227(5)$ & $0.0279(6)$ & $0.0354(6)$ & $0.0041(5)$ & $-0.0043(5)$ & $0.0031(6)$ \\
$\mathrm{O} 11$ & $0.080(3)$ & $0.112(4)$ & $0.097(4)$ & $-0.053(3)$ & $-0.026(3)$ & $0.032(3)$ \\
$\mathrm{O} 12$ & $0.058(2)$ & $0.044(2)$ & $0.160(4)$ & $0.010(2)$ & $0.000(2)$ & $0.016(3)$ \\
$\mathrm{O} 13$ & $0.053(2)$ & $0.061(3)$ & $0.154(4)$ & $-0.013(2)$ & $0.037(3)$ & $-0.001(3)$ \\
$\mathrm{O} 21$ & $0.106(4)$ & $0.025(3)$ & $0.129(5)$ & 0.000 & $0.077(4)$ & 0.000 \\
$\mathrm{O} 22$ & $0.070(3)$ & $0.100(3)$ & $0.105(3)$ & $0.054(3)$ & $0.041(3)$ & $0.036(3)$ \\
$\mathrm{N} 1$ & $0.0195(17)$ & $0.0221(18)$ & $0.027(2)$ & $0.0040(14)$ & $0.0037(16)$ & $0.0043(16)$ \\
$\mathrm{N} 2$ & $0.0240(18)$ & $0.0266(19)$ & $0.033(2)$ & $0.0020(15)$ & $0.0058(16)$ & $-0.0011(16)$ \\
$\mathrm{N} 3$ & $0.0243(18)$ & $0.0279(19)$ & $0.030(2)$ & $-0.0017(16)$ & $0.0012(16)$ & $0.0009(18)$ \\
$\mathrm{N} 11$ & $0.030(2)$ & $0.048(3)$ & $0.114(5)$ & $-0.015(2)$ & $0.004(3)$ & $-0.004(4)$ \\
$\mathrm{N} 21$ & $0.032(3)$ & $0.037(3)$ & $0.041(4)$ & 0.000 & $0.005(3)$ & 0.000 \\
$\mathrm{C} 1$ & $0.017(2)$ & $0.018(2)$ & $0.034(3)$ & $0.0004(16)$ & $0.0019(19)$ & $0.000(2)$ \\
$\mathrm{C} 2$ & $0.017(2)$ & $0.018(2)$ & $0.030(3)$ & $0.0008(16)$ & $0.0046(19)$ & $0.005(2)$ \\
$\mathrm{C} 3$ & $0.034(2)$ & $0.029(2)$ & $0.031(3)$ & $0.0115(19)$ & $0.016(2)$ & $0.006(2)$ \\
$\mathrm{C} 4$ & $0.022(2)$ & $0.035(3)$ & $0.031(3)$ & $0.0032(19)$ & $0.0019(19)$ & $0.000(2)$ \\
$\mathrm{C} 5$ & $0.033(3)$ & $0.043(3)$ & $0.082(4)$ & $0.010(2)$ & $0.014(3)$ & $-0.001(3)$ \\
$\mathrm{C} 6$ & $0.026(3)$ & $0.062(4)$ & $0.098(5)$ & $-0.002(3)$ & $0.012(3)$ & $-0.002(3)$ \\
$\mathrm{C} 7$ & $0.036(3)$ & $0.050(3)$ & $0.070(4)$ & $-0.010(2)$ & $0.008(3)$ & $-0.009(3)$ \\
$\mathrm{C} 8$ & $0.037(3)$ & $0.034(3)$ & $0.053(3)$ & $0.001(2)$ & $0.010(2)$ & $0.000(2)$ \\
$\mathrm{C} 9$ & $0.026(2)$ & $0.023(2)$ & $0.034(3)$ & $0.0067(18)$ & $0.000(2)$ & $0.000(2)$ \\
$\mathrm{C} 11$ & $0.041(3)$ & $0.032(3)$ & $0.048(3)$ & $-0.005(2)$ & $-0.003(2)$ & $0.003(2)$ \\
$\mathrm{C} 12$ & $0.048(3)$ & $0.044(3)$ & $0.072(4)$ & $-0.015(3)$ & $-0.020(3)$ & $0.006(3)$ \\
$\mathrm{C} 13$ & $0.034(3)$ & $0.054(4)$ & $0.100(5)$ & $-0.007(3)$ & $-0.026(3)$ & $0.018(3)$ \\
$\mathrm{C} 14$ & $0.032(3)$ & $0.038(3)$ & $0.074(4)$ & $0.006(2)$ & $-0.010(2)$ & $0.014(3)$ \\
$\mathrm{C} 15$ & $0.025(2)$ & $0.030(2)$ & $0.028(3)$ & $0.0002(19)$ & $0.0030(19)$ & $0.006(2)$ \\
& & & & &
\end{tabular}

Geometric parameters $\left(\AA,^{\circ}\right)$

\begin{tabular}{llll}
\hline $\mathrm{Ag} 1-\mathrm{N} 1$ & $2.578(3)$ & $\mathrm{N} 21-\mathrm{O} 22^{\mathrm{iii}}$ & $1.200(4)$ \\
$\mathrm{Ag} 1-\mathrm{N} 2^{\mathrm{i}}$ & $2.267(3)$ & $\mathrm{C} 1-\mathrm{C} 2$ & $1.384(5)$ \\
$\mathrm{Ag} 1-\mathrm{S} 1$ & $2.7943(13)$ & $\mathrm{C} 1-\mathrm{C} 3$ & $1.512(5)$ \\
$\mathrm{Ag} 1-\mathrm{S} 2^{\mathrm{ii}}$ & $2.6010(11)$ & $\mathrm{C} 2-\mathrm{N} 1^{\mathrm{ii}}$ & $1.331(5)$ \\
$\mathrm{Ag} 1-\mathrm{O} 11$ & $\mathrm{C} 2-\mathrm{C} 9$ & $1.523(5)$ \\
$\mathrm{Ag} 1-\mathrm{O} 13$ & $2.700(5)$ & $\mathrm{C} 3-\mathrm{H} 3 \mathrm{~A}$ & 0.9800 \\
$\mathrm{Ag} 2-\mathrm{N} 3$ & $2.752(5)$ & $\mathrm{C} 3-\mathrm{H} 3 \mathrm{~B}$ & 0.9800 \\
$\mathrm{Ag} 2-\mathrm{N} 3$ & $\mathrm{iii}$ & $\mathrm{C} 4-\mathrm{C} 5$ & $1.379(6)$ \\
$\mathrm{Ag} 2-\mathrm{O} 21$ & $2.208(3)$ & $\mathrm{C} 5-\mathrm{C} 6$ & $1.382(6)$ \\
$\mathrm{S} 1-\mathrm{C} 4$ & $2.208(3)$ & $\mathrm{C} 5-\mathrm{H} 5$ & 0.9400 \\
$\mathrm{~S} 1-\mathrm{C} 3$ & $2.567(5)$ & $\mathrm{C} 6-\mathrm{C} 7$ & $1.368(6)$ \\
$\mathrm{S} 2-\mathrm{C} 15$ & $1.770(4)$ & $\mathrm{C} 6-\mathrm{H} 6$ & 0.9400
\end{tabular}




\begin{tabular}{|c|c|c|c|}
\hline $\mathrm{S} 2-\mathrm{C} 9$ & $1.798(4)$ & $\mathrm{C} 7-\mathrm{C} 8$ & $1.353(6)$ \\
\hline $\mathrm{S} 2-\mathrm{Ag} 1^{\mathrm{ii}}$ & $2.6011(11)$ & $\mathrm{C} 7-\mathrm{H} 7$ & 0.9400 \\
\hline $\mathrm{O} 11-\mathrm{N} 11$ & $1.255(6)$ & $\mathrm{C} 8-\mathrm{H} 8$ & 0.9400 \\
\hline $\mathrm{O} 12-\mathrm{N} 11$ & $1.208(5)$ & $\mathrm{C} 9-\mathrm{H} 9 \mathrm{~A}$ & 0.9800 \\
\hline $\mathrm{O} 13-\mathrm{N} 11$ & $1.239(6)$ & С9-H9B & 0.9800 \\
\hline $\mathrm{O} 21-\mathrm{N} 21$ & $1.223(6)$ & $\mathrm{C} 11-\mathrm{C} 12$ & $1.360(6)$ \\
\hline $\mathrm{O} 22-\mathrm{N} 21$ & $1.200(4)$ & $\mathrm{C} 11-\mathrm{H} 11$ & 0.9400 \\
\hline $\mathrm{N} 1-\mathrm{C} 2^{\mathrm{ii}}$ & $1.331(5)$ & $\mathrm{C} 12-\mathrm{C} 13$ & $1.377(6)$ \\
\hline $\mathrm{N} 1-\mathrm{C} 1$ & $1.332(5)$ & $\mathrm{C} 12-\mathrm{H} 12$ & 0.9400 \\
\hline $\mathrm{N} 2-\mathrm{C} 4$ & $1.339(5)$ & $\mathrm{C} 13-\mathrm{C} 14$ & $1.374(6)$ \\
\hline $\mathrm{N} 2-\mathrm{C} 8$ & $1.351(5)$ & $\mathrm{C} 13-\mathrm{H} 13$ & 0.9400 \\
\hline $\mathrm{N} 2-\mathrm{Ag} 1^{\mathrm{i}}$ & $2.266(3)$ & $\mathrm{C} 14-\mathrm{C} 15$ & $1.372(6)$ \\
\hline $\mathrm{N} 3-\mathrm{C} 15$ & $1.343(5)$ & $\mathrm{C} 14-\mathrm{H} 14$ & 0.9400 \\
\hline $\mathrm{N} 3-\mathrm{C} 11$ & $1.345(5)$ & & \\
\hline $\mathrm{N} 1-\mathrm{Ag} 1-\mathrm{N} 2^{\mathrm{i}}$ & $155.31(11)$ & $\mathrm{N} 1^{\mathrm{ii}}-\mathrm{C} 2-\mathrm{C} 9$ & $118.4(3)$ \\
\hline $\mathrm{S} 1-\mathrm{Ag} 1-\mathrm{S} 2^{\mathrm{ii}}$ & $122.71(3)$ & $\mathrm{C} 1-\mathrm{C} 2-\mathrm{C} 9$ & $120.4(4)$ \\
\hline $\mathrm{S} 1-\mathrm{Ag} 1-\mathrm{N} 1$ & $68.98(7)$ & $\mathrm{C} 1-\mathrm{C} 3-\mathrm{S} 1$ & $117.4(3)$ \\
\hline $\mathrm{S} 1-\mathrm{Ag} 1-\mathrm{N} 2^{\mathrm{i}}$ & $96.92(8)$ & $\mathrm{C} 1-\mathrm{C} 3-\mathrm{H} 3 \mathrm{~A}$ & 107.9 \\
\hline $\mathrm{S} 2^{\mathrm{ii}}-\mathrm{Ag} 1-\mathrm{N} 1$ & $70.29(7)$ & $\mathrm{S} 1-\mathrm{C} 3-\mathrm{H} 3 \mathrm{~A}$ & 107.9 \\
\hline $\mathrm{S} 2^{\mathrm{ii}}-\mathrm{Ag} 1-\mathrm{N} 2^{\mathrm{i}}$ & $133.03(8)$ & $\mathrm{C} 1-\mathrm{C} 3-\mathrm{H} 3 \mathrm{~B}$ & 107.9 \\
\hline $\mathrm{S} 1-\mathrm{Ag} 1-\mathrm{O} 11$ & $122.18(10)$ & $\mathrm{S} 1-\mathrm{C} 3-\mathrm{H} 3 \mathrm{~B}$ & 107.9 \\
\hline $\mathrm{S} 1-\mathrm{Ag} 1-\mathrm{O} 13$ & $79.78(10)$ & $\mathrm{H} 3 \mathrm{~A}-\mathrm{C} 3-\mathrm{H} 3 \mathrm{~B}$ & 107.2 \\
\hline $\mathrm{S} 22^{\mathrm{ii}}-\mathrm{Ag} 1-\mathrm{O} 11$ & $81.18(10)$ & $\mathrm{N} 2-\mathrm{C} 4-\mathrm{C} 5$ & $122.4(4)$ \\
\hline $\mathrm{S} 2{ }^{\mathrm{ii}}-\mathrm{Ag} 1-\mathrm{O} 13$ & $120.26(10)$ & $\mathrm{N} 2-\mathrm{C} 4-\mathrm{S} 1$ & $112.5(3)$ \\
\hline $\mathrm{O} 11-\mathrm{Ag} 1-\mathrm{N} 1$ & $73.76(11)$ & $\mathrm{C} 5-\mathrm{C} 4-\mathrm{S} 1$ & $125.1(3)$ \\
\hline $\mathrm{O} 11-\mathrm{Ag} 1-\mathrm{N} 2^{\mathrm{i}}$ & 99.33 (12) & $\mathrm{C} 4-\mathrm{C} 5-\mathrm{C} 6$ & $118.2(4)$ \\
\hline $\mathrm{O} 13-\mathrm{Ag} 1-\mathrm{N} 1$ & $69.73(11)$ & $\mathrm{C} 4-\mathrm{C} 5-\mathrm{H} 5$ & 120.9 \\
\hline $\mathrm{O} 13-\mathrm{Ag} 1-\mathrm{N} 2^{\mathrm{i}}$ & $88.28(12)$ & $\mathrm{C} 6-\mathrm{C} 5-\mathrm{H} 5$ & 120.9 \\
\hline $\mathrm{O} 11-\mathrm{Ag} 1-\mathrm{O} 13$ & $45.99(14)$ & $\mathrm{C} 7-\mathrm{C} 6-\mathrm{C} 5$ & $119.7(4)$ \\
\hline $\mathrm{N} 3-\mathrm{Ag} 2-\mathrm{N} 3^{\mathrm{iii}}$ & $175.41(12)$ & $\mathrm{C} 7-\mathrm{C} 6-\mathrm{H} 6$ & 120.1 \\
\hline $\mathrm{O} 21-\mathrm{Ag} 2-\mathrm{N} 3$ & $92.30(9)$ & $\mathrm{C} 5-\mathrm{C} 6-\mathrm{H} 6$ & 120.1 \\
\hline $\mathrm{O} 21-\mathrm{Ag} 2-\mathrm{N} 3^{\mathrm{iii}}$ & $92.30(9)$ & $\mathrm{C} 8-\mathrm{C} 7-\mathrm{C} 6$ & $118.8(4)$ \\
\hline $\mathrm{C} 4-\mathrm{S} 1-\mathrm{C} 3$ & $103.19(19)$ & $\mathrm{C} 8-\mathrm{C} 7-\mathrm{H} 7$ & 120.6 \\
\hline $\mathrm{C} 4-\mathrm{S} 1-\mathrm{Ag} 1$ & $113.84(14)$ & $\mathrm{C} 6-\mathrm{C} 7-\mathrm{H} 7$ & 120.6 \\
\hline $\mathrm{C} 3-\mathrm{S} 1-\mathrm{Ag} 1$ & $98.68(14)$ & $\mathrm{N} 2-\mathrm{C} 8-\mathrm{C} 7$ & $123.1(4)$ \\
\hline $\mathrm{C} 15-\mathrm{S} 2-\mathrm{C} 9$ & $104.50(18)$ & $\mathrm{N} 2-\mathrm{C} 8-\mathrm{H} 8$ & 118.4 \\
\hline $\mathrm{C} 15-\mathrm{S} 2-\mathrm{Ag} 1^{\mathrm{ii}}$ & $98.56(13)$ & $\mathrm{C} 7-\mathrm{C} 8-\mathrm{H} 8$ & 118.4 \\
\hline $\mathrm{C} 9-\mathrm{S} 2-\mathrm{Ag} 1^{\mathrm{ii}}$ & $102.31(13)$ & $\mathrm{C} 2-\mathrm{C} 9-\mathrm{S} 2$ & $116.1(3)$ \\
\hline $\mathrm{N} 21-\mathrm{O} 21-\mathrm{Ag} 2$ & 180.0 & $\mathrm{C} 2-\mathrm{C} 9-\mathrm{H} 9 \mathrm{~A}$ & 108.3 \\
\hline $\mathrm{C} 2{ }^{\mathrm{ii}}-\mathrm{N} 1-\mathrm{C} 1$ & $118.2(3)$ & $\mathrm{S} 2-\mathrm{C} 9-\mathrm{H} 9 \mathrm{~A}$ & 108.3 \\
\hline $\mathrm{C} 22^{\mathrm{ii}}-\mathrm{N} 1-\mathrm{Ag} 1$ & $116.4(3)$ & $\mathrm{C} 2-\mathrm{C} 9-\mathrm{H} 9 \mathrm{~B}$ & 108.3 \\
\hline $\mathrm{C} 1-\mathrm{N} 1-\mathrm{Ag} 1$ & $118.0(2)$ & $\mathrm{S} 2-\mathrm{C} 9-\mathrm{H} 9 \mathrm{~B}$ & 108.3 \\
\hline $\mathrm{C} 4-\mathrm{N} 2-\mathrm{C} 8$ & $117.6(3)$ & $\mathrm{H} 9 \mathrm{~A}-\mathrm{C} 9-\mathrm{H} 9 \mathrm{~B}$ & 107.4 \\
\hline $\mathrm{C} 4-\mathrm{N} 2-\mathrm{Ag} 1^{\mathrm{i}}$ & $125.4(2)$ & $\mathrm{N} 3-\mathrm{C} 11-\mathrm{C} 12$ & $122.7(4)$ \\
\hline $\mathrm{C} 8-\mathrm{N} 2-\mathrm{Ag} 1^{\mathrm{i}}$ & $116.4(3)$ & $\mathrm{N} 3-\mathrm{C} 11-\mathrm{H} 11$ & 118.6 \\
\hline $\mathrm{C} 15-\mathrm{N} 3-\mathrm{C} 11$ & $117.8(3)$ & $\mathrm{C} 12-\mathrm{C} 11-\mathrm{H} 11$ & 118.6 \\
\hline $\mathrm{C} 15-\mathrm{N} 3-\mathrm{Ag} 2$ & $121.9(3)$ & $\mathrm{C} 11-\mathrm{C} 12-\mathrm{C} 13$ & $118.5(4)$ \\
\hline
\end{tabular}




\begin{tabular}{|c|c|c|c|}
\hline $\mathrm{C} 11-\mathrm{N} 3-\mathrm{Ag} 2$ & $119.7(3)$ & $\mathrm{C} 11-\mathrm{C} 12-\mathrm{H} 12$ & 120.8 \\
\hline $\mathrm{O} 12-\mathrm{N} 11-\mathrm{O} 13$ & $121.3(6)$ & $\mathrm{C} 13-\mathrm{C} 12-\mathrm{H} 12$ & 120.8 \\
\hline $\mathrm{O} 12-\mathrm{N} 11-\mathrm{O} 11$ & $121.5(6)$ & $\mathrm{C} 14-\mathrm{C} 13-\mathrm{C} 12$ & $120.3(4)$ \\
\hline $\mathrm{O} 13-\mathrm{N} 11-\mathrm{O} 11$ & $117.2(6)$ & $\mathrm{C} 14-\mathrm{C} 13-\mathrm{H} 13$ & 119.9 \\
\hline $\mathrm{O} 22-\mathrm{N} 21-\mathrm{O} 22^{\mathrm{iii}}$ & $122.4(6)$ & $\mathrm{C} 12-\mathrm{C} 13-\mathrm{H} 13$ & 119.9 \\
\hline $\mathrm{O} 22-\mathrm{N} 21-\mathrm{O} 21$ & $118.8(3)$ & $\mathrm{C} 15-\mathrm{C} 14-\mathrm{C} 13$ & $117.7(4)$ \\
\hline 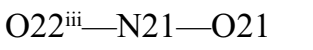 & $118.8(3)$ & $\mathrm{C} 15-\mathrm{C} 14-\mathrm{H} 14$ & 121.1 \\
\hline $\mathrm{N} 1-\mathrm{C} 1-\mathrm{C} 2$ & $120.7(3)$ & $\mathrm{C} 13-\mathrm{C} 14-\mathrm{H} 14$ & 121.1 \\
\hline $\mathrm{N} 1-\mathrm{C} 1-\mathrm{C} 3$ & $120.1(3)$ & $\mathrm{N} 3-\mathrm{C} 15-\mathrm{C} 14$ & $123.0(4)$ \\
\hline $\mathrm{C} 2-\mathrm{C} 1-\mathrm{C} 3$ & $119.2(4)$ & $\mathrm{N} 3-\mathrm{C} 15-\mathrm{S} 2$ & $111.5(3)$ \\
\hline $\mathrm{N} 1{ }^{\mathrm{ii}}-\mathrm{C} 2-\mathrm{C} 1$ & $121.1(4)$ & $\mathrm{C} 14-\mathrm{C} 15-\mathrm{S} 2$ & $125.5(3)$ \\
\hline $\mathrm{C} 2{ }^{\mathrm{ii}}-\mathrm{N} 1-\mathrm{C} 1-\mathrm{C} 2$ & $1.2(6)$ & $\mathrm{C} 5-\mathrm{C} 6-\mathrm{C} 7-\mathrm{C} 8$ & $0.0(8)$ \\
\hline $\mathrm{Ag} 1-\mathrm{N} 1-\mathrm{C} 1-\mathrm{C} 2$ & $150.0(3)$ & $\mathrm{C} 4-\mathrm{N} 2-\mathrm{C} 8-\mathrm{C} 7$ & $-0.8(6)$ \\
\hline $\mathrm{C} 2{ }^{\mathrm{ii}}-\mathrm{N} 1-\mathrm{C} 1-\mathrm{C} 3$ & $-177.7(3)$ & $\mathrm{Ag} 1{ }^{\mathrm{i}}-\mathrm{N} 2-\mathrm{C} 8-\mathrm{C} 7$ & $171.1(4)$ \\
\hline $\mathrm{Ag} 1-\mathrm{N} 1-\mathrm{C} 1-\mathrm{C} 3$ & $-28.9(4)$ & $\mathrm{C} 6-\mathrm{C} 7-\mathrm{C} 8-\mathrm{N} 2$ & $1.4(8)$ \\
\hline $\mathrm{N} 1-\mathrm{C} 1-\mathrm{C} 2-\mathrm{N} 1^{\mathrm{ii}}$ & $-1.3(6)$ & $\mathrm{N} 1{ }^{i i}-\mathrm{C} 2-\mathrm{C} 9-\mathrm{S} 2$ & $-2.7(5)$ \\
\hline $\mathrm{C} 3-\mathrm{C} 1-\mathrm{C} 2-\mathrm{N} 1^{\mathrm{ii}}$ & $177.7(3)$ & $\mathrm{C} 1-\mathrm{C} 2-\mathrm{C} 9-\mathrm{S} 2$ & $177.3(3)$ \\
\hline $\mathrm{N} 1-\mathrm{C} 1-\mathrm{C} 2-\mathrm{C} 9$ & $178.7(3)$ & $\mathrm{C} 15-\mathrm{S} 2-\mathrm{C} 9-\mathrm{C} 2$ & $-72.0(3)$ \\
\hline $\mathrm{C} 3-\mathrm{C} 1-\mathrm{C} 2-\mathrm{C} 9$ & $-2.3(5)$ & $\mathrm{Ag} 1{ }^{\mathrm{ii}}-\mathrm{S} 2-\mathrm{C} 9-\mathrm{C} 2$ & $30.3(3)$ \\
\hline $\mathrm{N} 1-\mathrm{C} 1-\mathrm{C} 3-\mathrm{S} 1$ & $-6.3(5)$ & $\mathrm{C} 15-\mathrm{N} 3-\mathrm{C} 11-\mathrm{C} 12$ & $-1.6(6)$ \\
\hline $\mathrm{C} 2-\mathrm{C} 1-\mathrm{C} 3-\mathrm{S} 1$ & $174.7(3)$ & $\mathrm{Ag} 2-\mathrm{N} 3-\mathrm{C} 11-\mathrm{C} 12$ & $169.3(4)$ \\
\hline $\mathrm{C} 4-\mathrm{S} 1-\mathrm{C} 3-\mathrm{C} 1$ & $-85.5(3)$ & $\mathrm{N} 3-\mathrm{C} 11-\mathrm{C} 12-\mathrm{C} 13$ & $0.9(7)$ \\
\hline $\mathrm{Ag} 1-\mathrm{S} 1-\mathrm{C} 3-\mathrm{C} 1$ & $31.6(3)$ & $\mathrm{C} 11-\mathrm{C} 12-\mathrm{C} 13-\mathrm{C} 14$ & $0.6(8)$ \\
\hline $\mathrm{C} 8-\mathrm{N} 2-\mathrm{C} 4-\mathrm{C} 5$ & $-1.0(6)$ & $\mathrm{C} 12-\mathrm{C} 13-\mathrm{C} 14-\mathrm{C} 15$ & $-1.2(8)$ \\
\hline $\mathrm{Ag} 1-\mathrm{N} 2-\mathrm{C} 4-\mathrm{C} 5$ & $-172.2(3)$ & $\mathrm{C} 11-\mathrm{N} 3-\mathrm{C} 15-\mathrm{C} 14$ & $0.9(6)$ \\
\hline $\mathrm{C} 8-\mathrm{N} 2-\mathrm{C} 4-\mathrm{S} 1$ & $178.2(3)$ & $\mathrm{Ag} 2-\mathrm{N} 3-\mathrm{C} 15-\mathrm{C} 14$ & $-169.7(3)$ \\
\hline $\mathrm{Ag} 1-\mathrm{N} 2-\mathrm{C} 4-\mathrm{S} 1$ & $7.1(4)$ & $\mathrm{C} 11-\mathrm{N} 3-\mathrm{C} 15-\mathrm{S} 2$ & $-179.5(3)$ \\
\hline $\mathrm{C} 3-\mathrm{S} 1-\mathrm{C} 4-\mathrm{N} 2$ & $-164.1(3)$ & $\mathrm{Ag} 2-\mathrm{N} 3-\mathrm{C} 15-\mathrm{S} 2$ & $9.8(4)$ \\
\hline $\mathrm{Ag} 1-\mathrm{S} 1-\mathrm{C} 4-\mathrm{N} 2$ & $90.0(3)$ & $\mathrm{C} 13-\mathrm{C} 14-\mathrm{C} 15-\mathrm{N} 3$ & $0.4(7)$ \\
\hline $\mathrm{C} 3-\mathrm{S} 1-\mathrm{C} 4-\mathrm{C} 5$ & $15.1(5)$ & $\mathrm{C} 13-\mathrm{C} 14-\mathrm{C} 15-\mathrm{S} 2$ & $-179.1(4)$ \\
\hline $\mathrm{Ag} 1-\mathrm{S} 1-\mathrm{C} 4-\mathrm{C} 5$ & $-90.8(4)$ & $\mathrm{C} 9-\mathrm{S} 2-\mathrm{C} 15-\mathrm{N} 3$ & $173.2(3)$ \\
\hline $\mathrm{N} 2-\mathrm{C} 4-\mathrm{C} 5-\mathrm{C} 6$ & $2.3(7)$ & $\mathrm{Ag} 1{ }^{\mathrm{ii}}-\mathrm{S} 2-\mathrm{C} 15-\mathrm{N} 3$ & $68.1(3)$ \\
\hline $\mathrm{S} 1-\mathrm{C} 4-\mathrm{C} 5-\mathrm{C} 6$ & $-176.8(4)$ & $\mathrm{C} 9-\mathrm{S} 2-\mathrm{C} 15-\mathrm{C} 14$ & $-7.2(4)$ \\
\hline $\mathrm{C} 4-\mathrm{C} 5-\mathrm{C} 6-\mathrm{C} 7$ & $-1.7(8)$ & $\mathrm{Ag} 1{ }^{\mathrm{ii}}-\mathrm{S} 2-\mathrm{C} 15-\mathrm{C} 14$ & $-112.4(4)$ \\
\hline
\end{tabular}

Symmetry codes: (i) $-x,-y+1,-z$; (ii) $-x+1 / 2,-y+1 / 2,-z$; (iii) $-x+1, y,-z+1 / 2$.

Hydrogen-bond geometry $\left(A,{ }^{\circ}\right)$

\begin{tabular}{lllll}
\hline$D-\mathrm{H} \cdots A$ & $D-\mathrm{H}$ & $\mathrm{H} \cdots A$ & $D \cdots A$ & $D-\mathrm{H} \cdots A$ \\
\hline $\mathrm{C} 11-\mathrm{H} 11 \cdots \mathrm{O} 21$ & 0.94 & 2.57 & $3.287(5)$ & 133 \\
$\mathrm{C} 3-\mathrm{H} 3 B \cdots \mathrm{O} 21^{\text {iv }}$ & 0.98 & 2.40 & $3.253(4)$ & 145 \\
$\mathrm{C} 3-\mathrm{H} 3 B \cdots \mathrm{O} 22^{\text {iv }}$ & 0.98 & 2.49 & $3.420(6)$ & 158 \\
$\mathrm{C} 7-\mathrm{H} 7 \cdots \mathrm{O} 13^{\mathrm{v}}$ & 0.94 & 2.51 & $3.268(6)$ & 138 \\
$\mathrm{C} 9-\mathrm{H} 9 A \cdots \mathrm{O} 22^{\text {iv }}$ & 0.98 & 2.32 & $3.291(6)$ & 171
\end{tabular}


supporting information

$\begin{array}{llllr}\mathrm{C} 12-\mathrm{H} 12 \cdots \mathrm{O} 11^{\mathrm{vi}} & 0.94 & 2.51 & 3.310(7) & 142 \\ \mathrm{C} 14-\mathrm{H} 14 \cdots \mathrm{O} 22^{\text {iv }} & 0.94 & 2.59 & 3.349(7) & 138\end{array}$

Symmetry codes: (iv) $x-1 / 2, y+1 / 2, z$; (v) $x+1 / 2, y+1 / 2, z$; (vi) $-x,-y,-z$. 